\title{
Pressão arterial e suas associações com atividade física e obesidade em adolescentes: uma revisão sistemática
}

\author{
Blood pressure and its association with physical activity \\ and obesity in adolescents: a systematic review
}

Victor Gonçalves Corrêa Neto ${ }^{1}$

Alexandre Palma ${ }^{1}$

${ }^{1}$ Escola de Educação Física e Desportos, Universidade Federal do Rio de Janeiro. Av. Carlos Chagas Filho 540, Cidade Universitária. 21.941-599 Rio de Janeiro RJ Brasil.

victorgcn@hotmail.com
Abstract In the light of the importance of the impacts that obesity and physical activity may have on blood pressure (BP) among adolescents, and the suggestion that abnormal pressure levels at young ages are maintained into more mature stages of these individuals' lives, the scope of this study is to conduct a systematic review of the associations between obesity and BP and between physical activity and BP among adolescents. The PubMed database was consulted to search for scientific articles relating to this topic and, after applying the appropriate inclusion and exclusion criteria, 30 articles were selected and analyzed. To assess the quality of articles Strengthening the Reporting of Observational Studies in Epidemiology was applied. The results suggest that despite the existence of hegemonic thinking to guide these relationships, BP and physical activity do not always have negative associations, and BP and obesity do not always have positive associations. The conclusion reached is that this situation illustrates the need for more in-depth reflection on these relationships before pre-established paradigms are passively accepted.

Key words Blood pressure, Exercise, Obesity, Adolescent
Resumo Diante da relevância do impacto que a obesidade e a atividade física podem possuir perante a pressão arterial (PA) em adolescentes, e da sugerida manutenção de cifras pressóricas alteradas em idades jovens para fases mais maduras da vida individuo, o presente estudo tem por objetivo revisar de forma sistemática a associação entre obesidade e PA, e entre atividade física e PA em adolescentes. A base de dados PubMed foi consultada para a busca de artigos científicos referentes ao tema, e após aplicar os devidos critérios de inclusão e exclusão, 30 artigos foram selecionados e analisados. Para avaliar a qualidade dos artigos, foi usado o Strengthening the Reporting of $\mathrm{Ob}$ servational Studies in Epidemiology. Os resultados sugerem que embora exista um pensamento hegemônico norteando tais relações, PA e atividade física nem sempre possuem associações negativas e PA e obesidade nem sempre mostram associações de cunho positivo. Conclui-se que tal quadro ilustra a necessidade de reflexões mais profundas sobre tais relações antes da aceitação passiva de paradigmas pré-estabelecidos.

Palavras chave Pressão arterial, Exercício, Obesidade, Adolescente 


\section{Introdução}

A hipertensão arterial sistêmica (HAS) é tida como relevante problema de saúde pública. Tal condição caracteriza-se quando da manutenção das cifras pressóricas elevadas de forma crônica acima dos pontos de corte tidos como de normalidade. Sua gênese tem premissa multifatorial e sua manifestação muitas vezes ocorre de forma assintomática. A literatura ainda advoga que tal enfermidade possui uma já bem estabelecida relação com aumento de risco cardiovascular ${ }^{1-4}$.

Evidências apontam na direção do aumento na prevalência de HAS na população adulta ${ }^{5}$. Tal quadro, contudo, parece não se limitar a indivíduos em faixas etárias mais maduras, mas também se estender a faixas etárias mais jovens ${ }^{6}$. Além disso, a alteração dos níveis pressóricos na infância parece ter interessante força de predição desses padrões fisiologicamente inadequados na fase madura do indivíduo. Estudos longitudinais ratificam essa informação $0^{7,8}$.

O crescente aumento nos casos de HAS não parece ser um fato isolado e independente, tal fenômeno parece vir ocorrendo em conjunto com um aumento da obesidade. Diferentes autores têm advogado que o ganho de peso em proporção patológica tem forte impacto sobre condições de comprometimento cardiovascular e doenças crônicas degenerativas. Que se destaque dentro de tal contexto a associação entre excesso de peso corporal e $\mathrm{HAS}^{9-13}$.

A associação entre HAS e obesidade não diz respeito somente a adultos, essa condição permanece também estabelecida em crianças, que uma vez acometidas por essa associação, parecem sustentar tal condição de forma longitudinal. Assim, a referida relação em idades mais jovens se apresenta com relevante força de predição da manutenção de tal contexto durante a maturidade do indivíduo ${ }^{14-17}$.

Um balanço calórico positivo se mostra diretamente associado à obesidade, e a atividade física parece ser componente de suma importância dentro da perspectiva de tal balanço. Com a suposta redução dos níveis de atividade física nas ultimas décadas dentre os mais jovens, acreditase na associação de tal fato com um aumento na prevalência de obesidade e por consequência das doenças e síndromes a ela relacionadas, tal como a $\mathrm{HAS}^{18,19}$.

Estratégias não farmacológicas parecem exercer significativo efeito no tratamento da $\mathrm{HAS}^{20}$. Seguindo tal linha de evidências, o American $\mathrm{Co}$ llege of Sports Medicine ${ }^{2}$, dá destaque aos efeitos positivos do exercício físico sobre os padrões pressóricos, ratificando o impacto benéfico no sentido profilático e de tratamento da atividade física sobre condições pressóricas alteradas, que parece ser algo já bem aceito no que tange às investigações na população adulta. Em adolescentes os resultados parecem um tanto quanto contraditórios $^{2}$, embora evidências sobre mecanismos fisiológicos já forneçam certa robustez e credibilidade na extrapolação das informações a respeito da eficácia da atividade física sobre os níveis pressóricos também de jovens e sublinhem o efeito terapêutico do exercício regular na HAS ${ }^{19}$.

Diante da relevância do impacto que a obesidade e a atividade física podem possuir perante a pressão arterial (PA) em adolescentes, o presente estudo tem por objetivo revisar de forma sistemática a associação entre obesidade e PA, e entre atividade física e PA em adolescentes.

\section{Método}

Foram levantados artigos da base de dados Pubmed até maio de 2012 com o intuito de recrutar investigações relevantes dentro dos critérios estabelecidos para compor a presente revisão.

As seguintes palavras chaves foram utilizadas em diferentes combinações na busca: hypertension, obesity, sedentary, physical activity, physical inactivity e adolescents. Os artigos encontrados tiveram seus títulos e resumos analisados dentro dos seguintes critérios de inclusão: a) mostrar de forma explícita que investigavam associações; b) ter amostra composta por indivíduos entre 10 e 19 anos, ou, se composta por faixas etárias maiores, conter estratificações dentro dos citados padrões etários. Entretanto, se as amostras ou estratificações passavam dentro da citada faixa etária e excediam ligeiramente para menos ou para mais, também foram considerados; c) estarem redigidos em língua inglesa; e d) serem estudos observacionais. Foram adotados os seguintes critérios de exclusão: a) artigos que tinham como objetivo investigar tais associações, mas envolviam outras síndromes e/ou doenças; b) estudos que analisavam o poder de uma intervenção; c) estudos de revisão. Os resumos que se enquadravam dentro de tais critérios, tiveram seus textos lidos de forma integral.

Os dois autores fizeram a leitura e seleção dos resumos e posterior leitura dos textos selecionados integralmente, quando considerados aptos consensualmente pelos dois os artigos eram incluídos como parte do estudo, quando não 
havia consenso, os autores se encontravam e discutiam o estudo até chegar a um parecer de comum acordo.

Para analisar a qualidade dos estudos, foram seguidos os parâmetros do Strengthening the Reporting of Observational Studies in Epidemio$\log y(\mathrm{STROBE})^{21}$. Cada um dos 22 itens que compõe o STROBE foram pontuados de zero a um pelos dois autores, a soma da pontuação de cada um dos 22 itens era considerada então, a nota final atribuída ao estudo pelo autor. Ao término do processo, a média da pontuação dos dois autores era considerada a nota final do estudo. Para facilitar a visualização dos resultados, os mesmos são ilustrados em valores percentuais, seguindo o modelo empregado por Mendes et al. ${ }^{22}$, no qual $50 \%$ foi o ponto de corte para considerar os estudos como de boa ou má qualidade.

As informações investigadas nos artigos que foram selecionados após passarem pelos critérios descritos acima foram: tamanho e representatividade da amostra; medidas de pressão arterial, obesidade e atividade física; e resultados.

\section{Resultados}

Durante a busca 439 artigos foram identificados. Destes, após a análise de seus títulos e resumos, dentro dos critérios preestabelecidos pela presente revisão, 32 investigações preencheram os requisitos necessários para fazer parte do presente estudo, tendo assim, seus textos lidos de forma integral. Durante a leitura na íntegra dos textos, outros dois estudos foram descartados, resultando em um total final de 30 artigos para compor a presente revisão. As principais razões para descarte de artigos foram estudos que não descreveram resultados a respeito de associações, que investigaram as associações em grupos com doenças ou características específicas, e estudos que extrapolaram em demasia a faixa etária alvo da revisão sem estratificar seus resultados (Figura 1$)$.

Em relação à avaliação da qualidade dos artigos, as notas dos autores não discordaram em relação à classificação baseada no ponto de corte de 50\% dos itens do STROBE. Apenas um artigo ficou abaixo de tal marca ${ }^{23}$. As Tabelas 1, 2 e 3 ilustram de forma descritiva as principais informações retiradas dos artigos com os resultados divididos em associações positivas, negativas e sem associação, respectivamente, bem como, a pontuação alcançada por cada estudo com base nos critérios do STROBE.
Dos 30 artigos, mais da metade deles (16), foram publicados na última metade da última década (2008-2012). Os outros 14 foram publicados até 2007.

Oito trabalhos foram realizados em países da América do Sul, sete em países da América do Norte, oito em países da Ásia, cinco em países da Europa e dois em país do continente Africano.

Dois estudos ${ }^{24,25}$ levantaram dados referentes apenas ao sexo feminino. Os demais estudos estenderam sua coleta de dados a ambos os sexos, porém, apenas seis dividiram seus resultados de associação por gênero dentro da faixa etária alvo da presente revisão ${ }^{26-31}$.

A faixa etária que teve o ponto de corte mais jovem entre os estudos foi cinco a dezessete anos ${ }^{32}$, enquanto a que teve o ponto de corte mais avançado cronologicamente foi quatorze a vinte anos $^{33}$, com uma variação no $n$ amostral de $82^{24}$ a $21.111^{34}$. Apenas um estudo teve seu desenho metodológico de caráter longitudinal ${ }^{25}$.

Uma investigação ${ }^{29}$ não descreve resultados a respeito de associações entre obesidade e PA, e 14 estudos não expõem as associações entre atividade física e $\mathrm{PA}^{11,12,23,26,30,32,35-42}$. Destaque-se que dos 14 estudos, cinco não se propuseram a fazer mensurações em relação à prática de atividades físicas $^{11,32,36,39,40}$.

\section{Obesidade e pressão arterial}

Com relação às associações entre obesidade e PA foram identificadas 28 positivas, quatro negativas e nove relatos da inexistência de associação entre as referidas variáveis (Quadro 1).

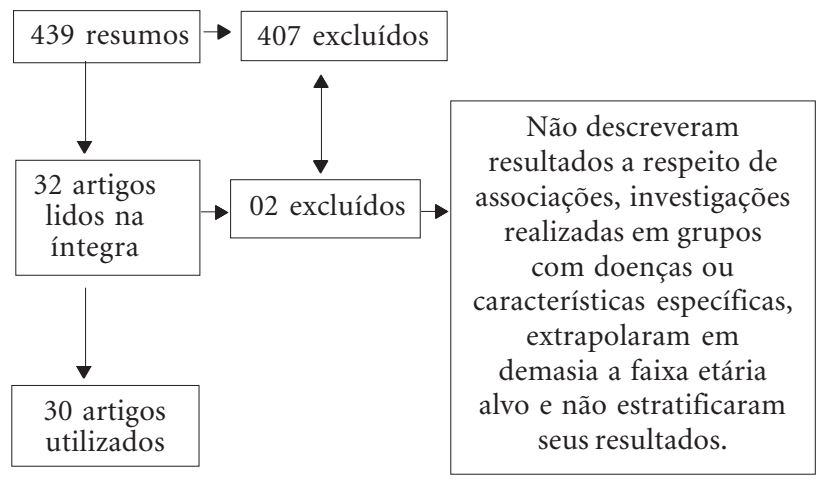

Figura 1. Fluxograma do processo de seleção dos artigos. 
Tabela 1. Estudos que ilustram associações positivas entre pressão arterial e obesidade.

\begin{tabular}{|c|c|c|c|c|}
\hline Referência & $\begin{array}{c}\text { Amostra / } \\
\text { Nação }\end{array}$ & Medidas & Resultados & $\begin{array}{l}\text { Pontuação } \\
\text { STROBE } \\
(\%)\end{array}$ \\
\hline $\begin{array}{c}\text { Moussa et al., } \\
1994^{42}\end{array}$ & $\begin{array}{c}\mathrm{n}=440 \\
\mathrm{M}=240 \\
\mathrm{~F}=200 \\
7 \text { a } 18 \text { anos } \\
\text { Emirados Árabes }\end{array}$ & $\begin{array}{c}\mathrm{PA}=\text { auscultatório } \\
\mathrm{OB}=\mathrm{IMC}, \text { razão } \\
\text { cintura quadril. } \\
\mathrm{AF}=\mathrm{NR}\end{array}$ & $\begin{array}{l}\text { Associação positiva entre IMC e } \\
\text { PAS }(\mathrm{p}<0,0004) \\
\text { Associação positiva entre IMC e } \\
\text { PAD }(\mathrm{p}<0,0001)\end{array}$ & $\begin{array}{l}13,12 \\
(59,6)\end{array}$ \\
\hline $\begin{array}{l}\text { Freedman et al., } \\
\qquad 1999^{32}\end{array}$ & $\begin{array}{c}\mathrm{n}=9167 \\
\mathrm{M}=4789 \\
\mathrm{~F}=4378 \\
5 \text { a } 17 \text { anos } \\
\text { Estados Unidos }\end{array}$ & $\begin{array}{c}\mathrm{PA}=\text { auscultatório } \\
\mathrm{OB}=\mathrm{IMC}, \text { dobras } \\
\text { cutâneas } \\
\text { subescapular e } \\
\text { tríceps, índice de } \\
\text { Rohrer }\end{array}$ & $\begin{array}{c}\text { Associação positiva entre IMC e } \\
\text { PAS (OR = 4,5; 3,6 - 5,8; } \\
\text { IC =95\%) } \\
\text { Associação positiva entre IMC e } \\
\text { PAD (OR =2,4; } 1,8-3 ; \\
\text { IC }=95 \%) \\
\text { Associação positiva entre índice } \\
\text { de Rohrer e PAS (OR = 4,5; } \\
\text { IC =95\%) } \\
\text { Associação positiva entre índice } \\
\text { de Rohrer e PAD (OR = 2,9; } \\
\text { IC = 95\%) } \\
\text { Associação positiva entre dobra } \\
\text { cutânea do tríceps e PAS } \\
\text { (OR = 3,4; IC = 95\%) } \\
\text { Associação positiva entre dobra } \\
\text { cutânea do tríceps e PAD } \\
\text { (OR = 2,6 IC = 95\%) }\end{array}$ & $\begin{array}{l}16,25 \\
(73,9)\end{array}$ \\
\hline Paterno, $2003^{43}$ & $\begin{array}{c}\mathrm{n}=2599 \\
\mathrm{M}=42 \% \\
\mathrm{~F}=58 \% \\
12 \text { a } 19 \text { anos } \\
\text { Argentina }\end{array}$ & $\begin{array}{c}\mathrm{PA}=\text { auscultatório } \\
\mathrm{OB}=\mathrm{IMC} \\
\mathrm{AF}=\text { índice } \\
\text { desenvolvido para } \\
\text { o estudo = } \\
\text { questionário } \\
\text { (outro) }\end{array}$ & $\begin{array}{c}\text { Associação positiva entre IMC e } \\
\text { HAS. (OR = 4,9; } 3,07-7,9 \\
\text { IC }=95 \%)\end{array}$ & $\begin{array}{l}16,37 \\
(74,4)\end{array}$ \\
\hline $\begin{array}{c}\text { Ribeiro et al, } \\
2004 .^{26}\end{array}$ & $\begin{array}{c}\mathrm{n}=1533 \\
\mathrm{M}=699 \\
\mathrm{~F}=762 \\
8 \text { a } 15 \text { anos } \\
\text { Portugal }\end{array}$ & $\begin{array}{c}\mathrm{PA}=\text { Automático } \\
\mathrm{OB}=\mathrm{IMC} \text {, dobras } \\
\text { cutâneas do tríceps } \\
\text { e subescapular. } \\
\text { AF = Questionário } \\
\text { (outro, MET, } \\
\text { calcula o Índice de } \\
\text { Atividade Física). }\end{array}$ & $\begin{array}{l}\text { Masc.: Associação positiva entre } \\
\text { percentual de gordura e PAS } \\
(\mathrm{r}=0,222 ; \mathrm{p}<0,001) \\
\text { Associação positiva entre alto } \\
\text { percentual de gordura e PA alta } \\
(\mathrm{OR}=2,6 ; 1,8-3,8 ; \mathrm{IC}=95 \%) \\
\text { Femin.: Associação positiva entre } \\
\text { percentual de gordura e PAS } \\
(\mathrm{r}=0,318 ; \mathrm{p}<0,001) \\
\text { Associação positiva entre } \\
\text { percentual de gordura e PAD } \\
(\mathrm{r}=0,249 ; \mathrm{p}<0,001) \\
\text { Associação positiva entre alto } \\
\text { percentual de gordura e PA alta } \\
(\mathrm{OR}=1,9 ; 1,3-2,8 ; \mathrm{IC}=95 \%)\end{array}$ & $\begin{array}{l}16,75 \\
(76,1)\end{array}$ \\
\hline
\end{tabular}


Tabela 1. continuação

\begin{tabular}{|c|c|c|c|c|}
\hline Referência & $\begin{array}{l}\text { Amostra / } \\
\text { Nação }\end{array}$ & Medidas & Resultados & $\begin{array}{c}\text { Pontuação } \\
\text { STROBE } \\
(\%)\end{array}$ \\
\hline Li et al., $2005^{36}$ & $\begin{array}{c}\mathrm{n}=1143 \\
\text { Ambos os sexos } \\
15 \text { a } 17 \text { anos } \\
\text { China }\end{array}$ & $\begin{array}{l}\text { PA - Auscultatório. } \\
\text { OB - IMC, CC. }\end{array}$ & $\begin{array}{l}\text { Associação positiva entre IMC e } \\
\text { HASist }(\mathrm{OR}=5 ; 1,5-16,4 \\
\text { p }<0,05) \\
\text { Associação positiva entre IMC e } \\
\text { HAD }(\mathrm{OR}=3,1 ; 1,2-8,1 \\
\mathrm{p}<0,05)\end{array}$ & $\begin{array}{r}15,37 \\
(69,9)\end{array}$ \\
\hline $\begin{array}{l}\text { Pileggi et al., } \\
2005^{37}\end{array}$ & $\begin{array}{l}\mathrm{n}=228 / 209 / 166 \\
\mathrm{M}=111 / 104 / 69 \\
\mathrm{~F}=117 / 105 / 97 \\
\text { Idade }=6-11 / \\
12-14 / 15-18 \\
\text { Itália }\end{array}$ & $\begin{array}{l}\mathrm{PA}=\text { auscultatório } \\
\mathrm{OB}=\mathrm{IMC} \\
\mathrm{AF}=\text { questionário } \\
\text { (outro) }\end{array}$ & $\begin{array}{l}\text { Associação positiva entre IMC e } \\
\text { PAS alta (OR = } 1,19 ; 1,1-1,29 ; \mathrm{p} \\
<0,001) \\
\text { Associação positiva entre IMC e } \\
\text { PAD alta (OR }=1,11 ; 1,04-1,2 ; \\
\quad \mathrm{p}=0,002)\end{array}$ & $\begin{array}{l}15,25 \\
(69,3)\end{array}$ \\
\hline $\begin{array}{l}\text { Monyeki et al., } \\
2006^{11}\end{array}$ & $\begin{array}{c}\mathrm{n}=1884 \\
\mathrm{M}=967 \\
\mathrm{~F}=917 \\
6 \text { a } 13 \text { anos } \\
\text { África do Sul }\end{array}$ & $\begin{array}{l}\mathrm{PA} \text { = automático } \\
\mathrm{OB}=\mathrm{IMC} \text {, dobras } \\
\text { cutâneas } \\
\text { suprailíacas, } \\
\text { subescapular, } \\
\text { tríceps e bíceps }\end{array}$ & $\begin{array}{l}\text { Associação positiva entre IMC e } \\
\operatorname{PAS}(\beta=0,003 ; \mathrm{p}=0,000) \\
\text { Associação positiva entre } \mathrm{S} / \mathrm{ST} \text { e } \\
\text { PAS }(\beta=0,03 ; \mathrm{p}=0,000) \\
\text { Associação positiva entre SS/SSBT } \\
\text { e PAS }(\beta=0,11 ; \mathrm{p}=0,000) \\
\text { Associação positiva entre }\end{array}$ & $\begin{array}{c}15 \\
(68,2)\end{array}$ \\
\hline $\begin{array}{l}\text { Ribeiro et al., } \\
2006^{12}\end{array}$ & $\begin{array}{c}\mathrm{n}=1450 \\
\mathrm{M}=47 \% \\
\mathrm{~F}=53 \% \\
6 \text { a } 18 \text { anos } \\
\text { Brasil }\end{array}$ & $\begin{array}{c}\mathrm{PA}=\text { não indica o } \\
\text { método } \\
\text { OB = IMC, dobras } \\
\text { cutâneas do } \\
\text { tríceps, } \\
\text { subescapular e } \\
\text { suprailíaca, CC e } \\
\text { do quadril. } \\
\text { AF = questionário } \\
\text { Outro. MET }\end{array}$ & $\begin{array}{l}\text { somatório das dobras cutâneas e } \\
\text { PAS }(\beta=0,10 ; p=0,000)\end{array}$ & $\begin{array}{l}16,62 \\
(75,5)\end{array}$ \\
\hline $\begin{array}{l}\text { Kelishadi et al., } \\
2006^{34}\end{array}$ & $\begin{array}{c}\mathrm{n}=21111 \\
\mathrm{M}=10253 \\
\mathrm{~F}=10858 \\
6 \text { a } 18 \text { anos } \\
\text { Irã }\end{array}$ & $\begin{array}{l}\mathrm{PA}=\text { auscultatório } \\
\mathrm{OB}=\mathrm{IMC}, \mathrm{CC}, \\
\text { circunferência do } \\
\text { quadril } \\
\mathrm{AF}=\text { questionário } \\
\text { (MET, boa relação } \\
\text { teste - reteste e } \\
\text { associado } \\
\text { significativamente } \\
\text { com o IPAQ e o } 7 \\
\text { day physical } \\
\text { activity) }\end{array}$ & 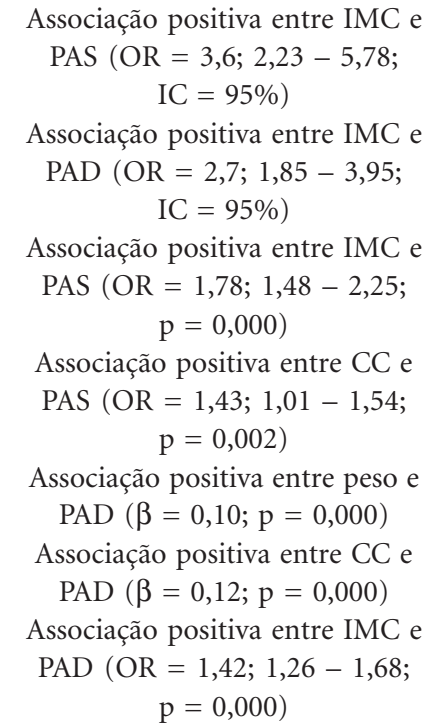 & $\begin{array}{l}16,5 \\
(75)\end{array}$ \\
\hline
\end{tabular}


Tabela 1. continuação

\begin{tabular}{|c|c|c|c|c|}
\hline Referência & $\begin{array}{c}\text { Amostra / } \\
\text { Nação }\end{array}$ & Medidas & Resultados & $\begin{array}{l}\text { Pontuação } \\
\text { STROBE } \\
(\%)\end{array}$ \\
\hline $\begin{array}{c}\text { Monego e } \\
\text { Jardim, } 2006^{44}\end{array}$ & $\begin{array}{c}\mathrm{n}=3169 \\
\mathrm{M}=1600 \\
\mathrm{~F}=1569 \\
7 \text { a } 14 \text { anos } \\
\text { Brasil }\end{array}$ & $\begin{array}{c}\mathrm{PA}=\text { auscultatório } \\
\mathrm{OB}=\mathrm{IMC} \\
\mathrm{AF}=\text { entrevista }\end{array}$ & $\begin{array}{l}\text { Associação positiva entre IMC e } \\
\text { níveis anormais de PA }(\mathrm{p}=0,01)\end{array}$ & $\begin{array}{c}15,5 \\
(70,5)\end{array}$ \\
\hline $\begin{array}{l}\text { Singh et al., } \\
2006^{45}\end{array}$ & $\begin{array}{c}\mathrm{n}=510 \\
\mathrm{M}=279 \\
\mathrm{~F}=231 \\
12 \text { a } 18 \text { anos } \\
\text { Índia }\end{array}$ & $\begin{array}{c}\text { PA = automático } \\
\text { OB = IMC } \\
\text { AF = questionário: } \\
\text { Global School- } \\
\text { Based Student } \\
\text { Health Survey } \\
\text { adaptado }\end{array}$ & $\begin{array}{c}\text { Associação positiva entre IMC e } \\
\text { PAS }(\beta=0,530 ; p=0,000) \\
\text { Associação positiva entre } \\
\text { obesidade e PAD }(\beta=2,169 \\
\text { p }=0,003)\end{array}$ & $\begin{array}{c}13,87 \\
(63)\end{array}$ \\
\hline $\begin{array}{l}\text { Forrest e Leeds, } \\
\qquad 2007^{35}\end{array}$ & $\begin{array}{c}\mathrm{n}=4109 \\
\mathrm{M}=51,8 \% \\
\mathrm{~F}=48,2 \% \\
12 \text { a } 19 \text { anos } \\
\text { Estados Unidos }\end{array}$ & $\begin{array}{c}\mathrm{PA}=\text { não relatado } \\
\mathrm{OB}=\mathrm{IMC} \text {, dobra } \\
\text { cutânea do tríceps, } \\
\quad \mathrm{CC} \\
\mathrm{AF}=\text { entrevista }\end{array}$ & $\begin{array}{c}\text { Associação positiva entre IMC e } \\
\text { PAS }(\mathrm{p}<0,001)\end{array}$ & $\begin{array}{l}13,12 \\
(59,6)\end{array}$ \\
\hline $\begin{array}{l}\text { Sugyiama et al., } \\
\qquad 2007^{46}\end{array}$ & $\begin{array}{c}\mathrm{n}=4508 \\
\mathrm{M}=50,9 \% \\
\mathrm{~F}=49,1 \% \\
12 \text { a } 19 \text { anos } \\
\text { Estados Unidos }\end{array}$ & $\begin{array}{c}\text { PA = auscultatório } \\
\text { OB = IMC } \\
\text { AF = entrevista } \\
\text { (outro) }\end{array}$ & $\begin{array}{l}\text { Associação positiva entre IMC e } \\
\text { PAS }(\beta=0,255 ; 0,218-0,292 ; \\
\text { p }<0,001)\end{array}$ & $\begin{array}{l}18,75 \\
(85,2)\end{array}$ \\
\hline $\begin{array}{l}\text { Silva e Lopes, } \\
2008^{27}\end{array}$ & $\begin{array}{c}\mathrm{n}=1570 \\
\mathrm{M}=808 \\
\mathrm{~F}=762 \\
7 \text { a } 12 \text { anos } \\
\text { Brasil }\end{array}$ & $\begin{array}{c}\mathrm{PA}=\text { auscultatório } \\
\mathrm{OB}=\mathrm{IMC} \text {, dobra } \\
\text { cutânea do tríceps } \\
\mathrm{AF}=\text { questionário } \\
\text { Typical Daily } \\
\text { Physical activities } \\
\text { and Meals (DAFA) }\end{array}$ & $\begin{array}{c}\text { Masc.: Associação positiva entre } \\
\text { excesso de peso e PA elevada } \\
(\mathrm{RP}=2,58 ; 1,61-4,13 ; \mathrm{p}<0,05) \\
\text { Associação positiva entre excesso } \\
\text { de peso e PAS }(\mathrm{RP}=1,99 ; 1,3 \text { - } \\
\text { 3,06; } \mathrm{p}<0,05) \\
\text { Femin: Associação positiva entre } \\
\text { excesso de peso e PA elevada } \\
(\mathrm{RP}=2,69 ; 1,97-3,68 ; \mathrm{p}<0,05) \\
\text { Associação positiva entre excesso } \\
\text { de peso e PAS }(\mathrm{RP}=2,09 ; \\
1,45-3,01 ; \mathrm{p}<0,05) \\
\text { Associação positiva entre excesso } \\
\text { de peso e PAD }(\mathrm{RP}=1,96 ; \\
1,41-2,75 ; \mathrm{p}<0,05)\end{array}$ & $\begin{array}{c}17 \\
(77,3)\end{array}$ \\
\hline $\begin{array}{l}\text { Saha et al., } \\
2008^{38}\end{array}$ & $\begin{array}{c}\mathrm{n}=1081 \\
\text { Ambos os sexos } \\
10 \text { a } 19 \text { anos } \\
\text { Índia }\end{array}$ & $\begin{array}{c}\text { PA - auscultatório } \\
\text { OB - IMC } \\
\text { AF - entrevista }\end{array}$ & $\begin{array}{l}\text { Associação positiva entre IMC e } \\
\text { PAS }(\mathrm{r}=0,753 ; \mathrm{p}=0,01) \\
\text { Associação positiva entre IMC e } \\
\text { PAD }(\mathrm{r}=0,689 ; \mathrm{p}=0,01)\end{array}$ & $\begin{array}{c}11,5 \\
(52,3)\end{array}$ \\
\hline $\begin{array}{l}\text { Salvadori et al., } \\
\qquad 2008^{39}\end{array}$ & $\begin{array}{c}\mathrm{n}=252 \\
\text { Ambos os sexos } \\
13 \text { a } 17 \text { anos } \\
\text { Canadá }\end{array}$ & $\begin{array}{l}\text { PA - manual e } \\
\text { automático } \\
\text { OB - IMC }\end{array}$ & $\begin{array}{c}\text { Associação positiva entre IMC e } \\
\text { HAS (OR }=5,9 ; 1,5-24,3 ; \\
\text { IC }=95 \%)\end{array}$ & $\begin{array}{c}18,25 \\
(83)\end{array}$ \\
\hline $\begin{array}{l}\text { Sporisevic et al., } \\
2009^{23}\end{array}$ & $\begin{array}{c}\mathrm{n}=214 \\
\mathrm{M}=112 \\
\mathrm{~F}=1027 \text { a } 15 \\
\text { anos } \\
\text { Bósnia }\end{array}$ & $\begin{aligned} \mathrm{PA}= & \text { Auscultatório } \\
\mathrm{OB}= & \mathrm{IMC} \\
\mathrm{AF}= & \text { Questionário } \\
& (\text { outro })\end{aligned}$ & $\begin{array}{c}\text { Associação positiva entre IMC e } \\
\text { HAS }(\mathrm{p}<0,05)\end{array}$ & $\begin{array}{l}10,62 \\
(48,3)\end{array}$ \\
\hline
\end{tabular}


Tabela 1. continuação

\begin{tabular}{|c|c|c|c|c|}
\hline Referência & $\begin{array}{l}\text { Amostra / } \\
\text { Nação }\end{array}$ & Medidas & Resultados & $\begin{array}{l}\text { Pontuação } \\
\text { STROBE } \\
(\%)\end{array}$ \\
\hline $\begin{array}{l}\text { Candido et al., } \\
2009^{47}\end{array}$ & $\begin{array}{c}\mathrm{n}=780 \\
\text { Ambos os sexos } \\
6 \text { a } 14 \text { anos } \\
\text { Brasil }\end{array}$ & $\begin{array}{l}\mathrm{PA}=\text { auscultatório } \\
\text { e automáticoOB }= \\
\mathrm{IMC}, \mathrm{CCAF}= \\
\text { entrevista (outro) }\end{array}$ & $\begin{array}{c}\text { Associação positiva entre IMC e } \\
\text { PA }(\mathrm{OR}=6,7 ; 2,6-16,5 ; \\
\mathrm{p}<0,001) \\
\text { Associação positiva entre CC e PA } \\
(\mathrm{OR}=7,8 ; 3,8-16,4 ; \mathrm{p}<0,001)\end{array}$ & $\begin{array}{l}16,12 \\
(73,3)\end{array}$ \\
\hline $\begin{array}{l}\text { Obarzanec et al., } \\
\qquad 2010^{25}\end{array}$ & $\begin{array}{c}\mathrm{n}=2368 \\
\mathrm{~F} \\
10 \text { a } 18 \text { anos } \\
\text { Estados Unidos }\end{array}$ & $\begin{array}{l}\mathrm{PA}=\text { auscultatório } \\
\mathrm{OB}=\mathrm{IMC}, \mathrm{CC} \\
\mathrm{AF}=\text { questionário } \\
\text { (Questionário } \\
\text { modelo de } \\
\text { atividades físicas } \\
\text { habituais) }\end{array}$ & $\begin{array}{l}\text { Associação positiva entre IMC e } \\
\text { desenvolvimento de HAS } \\
(\mathrm{p}<0,001) \\
\text { Associação positiva entre CC e } \\
\text { desenvolvimento de HAS } \\
(\mathrm{p}=0,029) .\end{array}$ & $\begin{array}{c}14,75 \\
(67)\end{array}$ \\
\hline $\begin{array}{l}\text { Yi-Choun Chou } \\
\text { e Jen-Sheng Pei, } \\
2010^{28}\end{array}$ & $\begin{array}{c}\mathrm{n}=558 \\
\mathrm{M}=284 \\
\mathrm{~F}=274 \\
12 \text { a } 18 \text { anos } \\
\text { Taiwan }\end{array}$ & $\begin{array}{c}\text { PA = automático } \\
\text { OB = IMC, CC } \\
\text { AF = Questionário }\end{array}$ & $\begin{array}{l}\text { Masc.: Associação positiva entre } \\
\text { IMC e PAS }(\mathrm{r}=0,57 ; \mathrm{p}<0,05) \\
\text { Associação positiva entre IMC e } \\
\text { PAD }(\mathrm{r}=0,428 ; \mathrm{p}<0,05) \\
\text { Femin.: Associação positiva entre } \\
\text { IMC e PAS }(\mathrm{r}=0,568 ; \mathrm{p}<0,05) \\
\text { Associação positiva entre IMC e } \\
\text { PAD ( } \mathrm{r}=0,519 ; \mathrm{p}<0,05)\end{array}$ & $\begin{array}{l}18,75 \\
(85,2)\end{array}$ \\
\hline $\begin{array}{l}\text { Burgos et al., } \\
2010^{40}\end{array}$ & $\begin{array}{c}\mathrm{n}=1666 \\
\mathrm{M}=873 \\
\mathrm{~F}=793 \\
7 \text { a } 17 \text { anos } \\
\text { Brasil }\end{array}$ & $\begin{array}{l}\mathrm{PA}=\text { auscultatório } \\
\mathrm{OB}=\mathrm{IMC} \text {, dobras } \\
\text { cutâneas do tríceps } \\
\text { e subescapular, CC } \\
\text { e circunferência do } \\
\text { quadril }\end{array}$ & $\begin{array}{l}\text { Associação positiva entre peso e } \\
\text { PAS }(\mathrm{r}=0,597 ; \mathrm{p}<0,05) \\
\text { Associação positiva entre IMC e } \\
\text { PAS }(\mathrm{r}=0,476 ; \mathrm{p}<0,05) \\
\text { Associação positiva entre CC e } \\
\text { PAS }(\mathrm{r}=0,498 ; \mathrm{p}<0,05) \\
\text { Associação positiva entre } \\
\text { somatório das dobras cutâneas e } \\
\text { PAS ( } \mathrm{r}=0,300 ; \mathrm{p}<0,05) \\
\text { Associação positiva entre } \\
\text { percentual de gordura corporal e } \\
\text { PAS }(\mathrm{r}=0,292 ; \mathrm{p}<0,05) \\
\text { Associação positiva entre peso e } \\
\text { PAD ( } \mathrm{r}=0,507 ; \mathrm{p}<0,05) \\
\text { Associação positiva entre IMC e } \\
\text { PAD ( } \mathrm{r}=0,413 ; \mathrm{p}<0,05) \\
\text { Associação positiva entre CC e } \\
\text { PAD }(\mathrm{r}=0,405 ; \mathrm{p}<0,05) \\
\text { Associação positiva entre } \\
\text { somatório das dobras cutâneas e } \\
\text { PAD ( } \mathrm{r}=0,269 ; \mathrm{p}<0,05) \\
\text { Associação positiva entre } \\
\text { percentual de gordura corporal e } \\
\text { PAD ( } \mathrm{r}=0,266 ; \mathrm{p}<0,05 \text { ) }\end{array}$ & $\begin{array}{l}16,37 \\
(74,4)\end{array}$ \\
\hline $\begin{array}{l}\text { Griz et al., } \\
2010^{33}\end{array}$ & $\begin{array}{c}\mathrm{n}=1825 \\
\mathrm{M}=40 \% \\
\mathrm{~F}=60 \% \\
14 \text { a } 20 \text { anos } \\
\text { Brasil }\end{array}$ & $\begin{array}{l}\text { PA = auscultatório } \\
\text { OB = IMC, CC } \\
\text { AF = questionário: } \\
\text { Global School- } \\
\text { Based Student } \\
\text { Health Survey }\end{array}$ & $\begin{array}{l}\text { Associação positiva entre CC e } \\
\text { HAS }(\mathrm{OR}=2,48 ; 2,49-4,87 \\
\qquad \mathrm{p}<0,001)\end{array}$ & $\begin{array}{l}15,75 \\
(71,6)\end{array}$ \\
\hline
\end{tabular}


Tabela 1. continuação

\begin{tabular}{|c|c|c|c|c|}
\hline Referência & $\begin{array}{l}\text { Amostra / } \\
\text { Nação }\end{array}$ & Medidas & Resultados & $\begin{array}{l}\text { Pontuação } \\
\text { STROBE } \\
(\%)\end{array}$ \\
\hline $\begin{array}{l}\text { Polderman et } \\
\text { al., } 2011^{30}\end{array}$ & $\begin{array}{c}\mathrm{n}=1002 \\
\mathrm{M}=442 \\
\mathrm{~F}=560 \\
12 \text { a } 17 \text { anos } \\
\text { Brasil }\end{array}$ & $\begin{array}{c}\text { PA - automático } \\
\text { OB - IMC } \\
\text { AF - questionário } \\
\text { (outro) }\end{array}$ & $\begin{array}{c}\text { Masc.: Associação positiva entre } \\
\text { IMC e PA (RP = 4,87; } \\
2,35-10,11 ; \mathrm{IC}=95 \%) \\
\text { Femin.: Associação positiva entre } \\
\text { IMC e PA (RP = 5,18; } \\
2,67-10,06 ; \mathrm{IC}=95 \%)\end{array}$ & $\begin{array}{l}17,62 \\
(80,1)\end{array}$ \\
\hline $\begin{array}{l}\text { Lazorick et al., } \\
\qquad 2011^{41}\end{array}$ & $\begin{array}{c}\mathrm{n}=1308 \\
\text { Ambos os sexos } \\
13 \text { a } 16 \text { anos } \\
\text { EUA }\end{array}$ & $\begin{array}{c}\mathrm{PA}=\text { registro } \\
\text { médico } \\
\mathrm{OB}=\mathrm{IMC} \\
\mathrm{AF}=\text { registro } \\
\text { médico }\end{array}$ & $\begin{array}{c}\text { Associação positiva entre IMC e } \\
\text { HAS }(\mathrm{p}<0,01)\end{array}$ & $\begin{array}{l}17,12 \\
(77,8)\end{array}$ \\
\hline $\begin{array}{l}\text { Gaya et al., } \\
2011^{48}\end{array}$ & $\begin{array}{c}\mathrm{n}=1075 \\
\mathrm{M}=503 \\
\mathrm{~F}=572 \\
8 \text { a } 17 \text { anos } \\
\text { Portugal }\end{array}$ & $\begin{array}{l}\mathrm{PA}=\text { automático } \\
\mathrm{OB}=\mathrm{IMC} \\
\mathrm{AF}=\text { questionário }\end{array}$ & $\begin{array}{c}\text { Associação positiva entre IMC e } \\
\text { PA alta - modelo com atividades } \\
\text { de competição esportiva - } \\
\text { (OR }=2,17 ; 1,56-3,02 ; \mathrm{p}<0,05) \\
\text { Associação positiva entre IMC e } \\
\text { PA alta - modelo com AF no } \\
\text { tempo de lazer - }(\mathrm{OR}=2,22 ; \\
1,59-3,09 ; \mathrm{p}<0,05)\end{array}$ & $\begin{array}{l}17,25 \\
(78,4)\end{array}$ \\
\hline $\begin{array}{l}\text { Leung et al., } \\
\qquad 2011^{49}\end{array}$ & $\begin{array}{c}\mathrm{n}=5901 \\
\mathrm{M}=2938 \\
\mathrm{~F}=2963 \\
\text { Média de } 15,2 \\
\text { anos de idade } \\
\text { China }\end{array}$ & $\begin{array}{c}\mathrm{PA} \text { = automático } \\
\mathrm{OB} \text { - IMC, CC } \\
\mathrm{AF} \text { - questionário } \\
\text { (outro) }\end{array}$ & $\begin{array}{l}\text { Associação positiva entre CC e } \\
\text { HAS }(\mathrm{OR}=2,378 ; 1,164-4,987 \\
\qquad \mathrm{p}=0,022)\end{array}$ & $\begin{array}{l}19,12 \\
(86,9)\end{array}$ \\
\hline $\begin{array}{l}\text { Hujova e } \\
\text { Lesniakova, } \\
2011^{50}\end{array}$ & $\begin{array}{c}\mathrm{n}=191 \\
\mathrm{M}=97 \\
\mathrm{~F}=94 \\
\text { Idade } 7-11 / 12-18 \\
\text { Eslováquia }\end{array}$ & $\begin{array}{l}\mathrm{PA}=\text { auscultatório } \\
\mathrm{OB}=\mathrm{IMC}, \text { dobras } \\
\text { cutâneas do tríceps } \\
\text { e subescapular, CC, } \\
\text { circunferência do } \\
\text { quadril. } \\
\mathrm{AF}=\text { questionário } \\
\text { (outro) }\end{array}$ & $\begin{array}{l}\text { Associação positiva entre IMC e } \\
\text { PAD ( } \mathrm{p}<0,05) \\
\text { Associação positiva entre IMC e } \\
\text { PAS ( } \mathrm{p}<0,05) \\
\text { Associação positiva entre razão } \\
\text { cintura quadril e PAD somente } \\
\text { em adolescentes }(\mathrm{r}=0,202)\end{array}$ & $\begin{array}{c}14 \\
(63,6)\end{array}$ \\
\hline $\begin{array}{l}\text { Aounallah - } \\
\text { Skhiri et al., } \\
\quad 2012^{31}\end{array}$ & $\begin{array}{c}\mathrm{n}=2870 \\
\mathrm{M}=1294 \\
\mathrm{~F}=1576 \\
15 \text { a } 19 \text { anos } \\
\text { Tunísia }\end{array}$ & $\begin{array}{c}\mathrm{PA}=\text { auscultatório } \\
\mathrm{OB}=\mathrm{IMC}, \mathrm{CC} \\
\mathrm{AF}=\text { entrevista }\end{array}$ & $\begin{array}{l}\text { Masc.: Associação positiva entre } \\
\text { IMC e HAS (OR = 3,5; } 1,4-8,9 ; \\
\text { p }=0,012) \\
\text { Associação positiva entre CC e } \\
\text { HAS (OR }=2,7 ; 1,1-6,6 ; \mathrm{p}= \\
0,0046) \\
\text { Femin.: Associação positiva entre } \\
\text { IMC e HAS (OR }=5,4 ; 2,2-13,4 ; \\
\text { p }=0,0017)\end{array}$ & $\begin{array}{c}17,37 \\
(79)\end{array}$ \\
\hline
\end{tabular}

* $\mathrm{M}$ = Sexo masculino, $\mathrm{F}$ = Sexo feminino, $\mathrm{PA}$ = pressão arterial, $\mathrm{OB}=$ obesidade, $\mathrm{AF}=$ atividade física, $\mathrm{PAS}$ = pressão arterial sistólica, PAD = pressão arterial diastólica, HÁS = hipertensão arterial sistêmica, HASist = hipertensão arterial sistólica, $\mathrm{HAD}=$ hipertensão arterial diastólica, $\mathrm{IMC}=$ índice de massa corporal; $\mathrm{CC}=$ circunferência da cintura, $\mathrm{S} / \mathrm{T}=$ subescapular/tríceps, $\mathrm{S} / \mathrm{ST}$ = subescapular/subescapular+tríceps, $\mathrm{SS} / \mathrm{SSBT}=$ subescapular+suprailíaca/subescapular+suprailíaca+bíceps+tríceps, NR = não relatado, $\mathrm{STROBE}=$ Strengthening the Reporting of Observational Studies in Epidemiology. 
Tabela 2. Estudos que ilustram associações negativas entre pressão arterial e atividade física e entre pressão arterial e obesidade.

\begin{tabular}{|c|c|c|c|c|}
\hline Referência & $\begin{array}{l}\text { Amostra / } \\
\text { Nação }\end{array}$ & Medidas & Resultados & $\begin{array}{l}\text { Pontuação } \\
\text { STROBE } \\
(\%)\end{array}$ \\
\hline Paterno, $2003^{43}$ & $\begin{array}{c}\mathrm{n}=2599 \\
\mathrm{M}=42 \% \\
\mathrm{~F}=58 \% \\
12 \text { a } 19 \text { anos } \\
\text { Argentina }\end{array}$ & $\begin{array}{c}\mathrm{PA}=\text { auscultatório } \\
\mathrm{OB}=\mathrm{IMC} \\
\mathrm{AF}=\text { índice } \\
\text { desenvolvido para } \\
\text { o estudo }= \\
\text { questionário } \\
\text { (outro) }\end{array}$ & $\begin{array}{c}\text { Associação negativa entre AF e } \\
\text { HAS (OR }=0,77 ; 0,59-0,99 ; \\
\text { IC }=95 \%)\end{array}$ & $\begin{array}{l}16,37 \\
(74,4)\end{array}$ \\
\hline $\begin{array}{l}\text { Kelishadi et al., } \\
2006^{34}\end{array}$ & $\begin{array}{c}\mathrm{n}=21111 \\
\mathrm{M}=10253 \\
\mathrm{~F}=10858 \\
6 \text { a } 18 \text { anos } \\
\text { Irã }\end{array}$ & $\begin{array}{c}\mathrm{PA}=\text { auscultatório } \\
\mathrm{OB}=\text { IMC, CC, } \\
\text { circunferência do } \\
\text { quadril } \\
\mathrm{AF}=\text { questionário } \\
\text { (MET, boa relação } \\
\text { teste - reteste e } \\
\text { associado } \\
\text { significativamente } \\
\text { com o IPAQ e o } 7 \\
\text { day physical } \\
\text { activity) }\end{array}$ & $\begin{array}{c}\text { Associação negativa entre AF } \\
\text { e PAS }(\beta=-0,19 ; \mathrm{p}= \\
0,042) . \text { Associação negativa } \\
\text { entre CQ e PAD }(\beta=-0,04 ; \mathrm{p} \\
=0,037) \text {.Associação negativa } \\
\text { entre AF e PAD([referência } \\
\text { ativos] OR }=1,71 ; 1,53- \\
1,95 ; \mathrm{p}=0,019)\end{array}$ & $\begin{array}{c}16 \\
(72,7)\end{array}$ \\
\hline $\begin{array}{l}\text { Forrest e Leeds, } \\
\quad 2007^{35}\end{array}$ & $\begin{array}{c}\mathrm{n}=4109 \\
\mathrm{M}=51,8 \% \\
\mathrm{~F}=48,2 \% \\
12 \text { a } 19 \text { anos } \\
\text { Estados Unidos }\end{array}$ & $\begin{array}{c}\mathrm{PA}=\text { não relatado } \\
\mathrm{OB}=\mathrm{IMC} \text {, dobra } \\
\text { cutânea do tríceps, } \\
\quad \mathrm{CC} \\
\mathrm{AF}=\text { entrevista }\end{array}$ & $\begin{array}{l}\text { Associação negativa entre } \\
\text { IMC e PAD }(p<0,01)\end{array}$ & $\begin{array}{l}13,12 \\
(59,6)\end{array}$ \\
\hline $\begin{array}{l}\text { Sugyiama et al., } \\
\qquad 2007^{46}\end{array}$ & $\begin{array}{c}\mathrm{n}=4508 \\
\mathrm{M}=50,9 \% \\
\mathrm{~F}=49,1 \% \\
12 \text { a } 19 \text { anos } \\
\text { Estados Unidos }\end{array}$ & $\begin{array}{c}\mathrm{PA}=\text { auscultatório } \\
\mathrm{OB}=\mathrm{IMC} \\
\mathrm{AF}=\text { entrevista } \\
\text { (outro) }\end{array}$ & $\begin{array}{l}\text { Associação negativa entre } \\
\text { atividade física vigorosa e } \\
\text { PAD }(\beta=-0,109 ; \\
-0,198-0,19 ; p=0,02) \\
\text { Associação negativa entre } \\
\text { IMC e PAD }(\beta=-0,073 ; \\
-0,107--0,038 ; p<0,01)\end{array}$ & $\begin{array}{l}18,75 \\
(85,2)\end{array}$ \\
\hline
\end{tabular}

continua

O número de associações é superior ao número de estudos, pois algumas investigações encontraram resultados discordantes no que diz respeito aos métodos empregados ou a diferentes gêneros estudados.

Todos os estudos encontraram, em alguma medida de obesidade, associação de cunho positivo com a PA, exceto Taylor et al. ${ }^{24}$, que utilizaram como método avaliador da obesidade o índice de massa corporal (IMC), e não encontraram associação de tal medida nem com pressão arterial sistólica (PAS) e nem com pressão arterial diastólica (PAD).

Além de Taylor et al. ${ }^{24}$, mais oito artigos constataram em algum momento a inexistência de associação entre obesidade e $\mathrm{PA}^{11,26,27,31,34,42,49,50}$.
Dos seis estudos que avaliaram separadamente as associações entre os diferentes gêneros, cinco deles se propuseram a investigar obesidade ${ }^{26-}$ 28,30,31, e três não encontraram associação significativa em alguma de suas medidas ${ }^{26,27,31}$. Ribeiro et al. ${ }^{26}$ não observaram associação entre percentual de gordura e PAD no sexo masculino, já Silva et al. ${ }^{27}$ não identificaram associação entre excesso de gordura corporal e PA elevada tanto no sexo masculino, quanto no feminino. Aounallahskhiri et al. ${ }^{31}$ não foram capazes de identificar associação significativa entre circunferência de cintura e HAS em meninas. Ainda, outros artigos levantados, analisaram sua amostra sem divisão por sexo, e ainda assim ilustraram a falta de associação significativa entre obesidade e pressão 
Tabela 2. continuação

\begin{tabular}{|c|c|c|c|c|}
\hline Referência & $\begin{array}{c}\text { Amostra / } \\
\text { Nação }\end{array}$ & Medidas & Resultados & $\begin{array}{l}\text { Pontuação } \\
\text { STROBE } \\
(\%)\end{array}$ \\
\hline $\begin{array}{l}\text { Burgos et al., } \\
\quad 2010^{40}\end{array}$ & $\begin{array}{c}\mathrm{n}=1666 \\
\mathrm{M}=873 \\
\mathrm{~F}=793 \\
7 \text { a } 17 \text { anos } \\
\text { Brasil }\end{array}$ & $\begin{array}{l}\text { PA = auscultatório } \\
\text { OB = IMC, dobras } \\
\text { cutâneas do tríceps } \\
\text { e subescapular, CC } \\
\text { e circunferência do } \\
\text { quadril }\end{array}$ & $\begin{array}{c}\text { Associação negativa entre razão } \\
\text { cintura quadril e PAS }(\mathrm{r}=-0,120 \text {; } \\
\mathrm{p}<0,05) \\
\text { Associação negativa entre razão } \\
\text { cintura quadril e PAD } \\
(\mathrm{r}=-0,146 ; \mathrm{p}<0,05)\end{array}$ & $\begin{array}{l}16,37 \\
(74,4)\end{array}$ \\
\hline $\begin{array}{l}\text { Griz et al., } \\
2010^{33}\end{array}$ & $\begin{array}{c}\mathrm{n}=1825 \\
\mathrm{M}=40 \% \\
\mathrm{~F}=60 \% \\
14 \text { a } 20 \text { anos } \\
\text { Brasil }\end{array}$ & $\begin{array}{l}\text { PA = auscultatório } \\
\text { OB = IMC, CC } \\
\text { AF = questionário: } \\
\text { Global School- } \\
\text { Based Student } \\
\text { Health Survey }\end{array}$ & $\begin{array}{l}\text { Associação negativa entre AF e } \\
\text { HAS (OR }=1,37 \text { [referência } \\
\text { ativos]; } 1,06-1,78 ; \mathrm{p}=0,015)\end{array}$ & $\begin{array}{l}15,75 \\
(71,6)\end{array}$ \\
\hline $\begin{array}{l}\text { Yoshinaga et al., } \\
\qquad 2011^{29}\end{array}$ & $\begin{array}{c}\mathrm{n}=755 \\
\mathrm{M}=331 \\
\mathrm{~F}=424,1 \\
5 \text { a } 18 \text { anos } \\
\text { Japão }\end{array}$ & $\begin{array}{c}\text { PA - automático } \\
\text { OB - IMC, CC } \\
\text { AF - Questionário } \\
\text { (outro) }\end{array}$ & $\begin{array}{l}\text { Masc.: Associação negativa entre } \\
\text { atividades físicas extracurriculares } \\
\text { e PAS }(\mathrm{p}<0,05)\end{array}$ & $\begin{array}{c}17 \\
(77,3)\end{array}$ \\
\hline $\begin{array}{l}\text { Gaya et al., } \\
2011^{48}\end{array}$ & $\begin{array}{c}\mathrm{n}=1075 \\
\mathrm{M}=503 \\
\mathrm{~F}=572 \\
8 \text { a } 17 \text { anos } \\
\text { Portugal }\end{array}$ & $\begin{array}{l}\mathrm{PA}=\text { automático } \\
\quad \mathrm{OB}=\mathrm{IMC} \\
\mathrm{AF}=\text { questionário }\end{array}$ & $\begin{array}{c}\text { Associação negativa entre AF no } \\
\text { tempo de lazer e PA alta } \\
(\mathrm{OR}=1,5[\text { referência ativo]; } \\
1,12-2,02 ; \mathrm{p}<0,05)\end{array}$ & $\begin{array}{l}17,25 \\
(78,4)\end{array}$ \\
\hline $\begin{array}{l}\text { Leung et al., } \\
2011^{49}\end{array}$ & $\begin{array}{c}\mathrm{n}=5901 \\
\mathrm{M}=2938 \\
\mathrm{~F}=2963 \\
\text { Média de } 15,2 \\
\text { anos de idade } \\
\text { China }\end{array}$ & $\begin{array}{l}\mathrm{PA}=\text { automático } \\
\text { OB - IMC, } \\
\text { circunferência da } \\
\quad \text { cintura } \\
\text { AF - questionário } \\
\quad \text { (outro) }\end{array}$ & $\begin{array}{c}\text { Associação negativa entre AF e } \\
\text { HAS }(\mathrm{OR}=0,232 \\
0,113-0,706 ; \mathrm{p}=0,007)\end{array}$ & $\begin{array}{l}19,12 \\
(86,9)\end{array}$ \\
\hline $\begin{array}{l}\text { Hujova e } \\
\text { Lesniakova, } \\
2011^{50}\end{array}$ & $\begin{array}{l}\qquad \mathrm{n}=191 \\
\mathrm{M}=97 \\
\mathrm{~F}=94 \\
\text { Idade } 7-11 / 12-18 \\
\text { Eslováquia }\end{array}$ & $\begin{array}{c}\mathrm{PA}=\text { auscultatório } \\
\mathrm{OB}=\mathrm{IMC}, \text { dobras } \\
\text { cutâneas do tríceps } \\
\text { e subescapular, CC, } \\
\text { circunferência do } \\
\text { quadril } \\
\mathrm{AF}=\text { questionário } \\
\text { (outro) }\end{array}$ & $\begin{array}{c}\text { Associação negativa entre } \\
\text { AF e PAD na população urbana } \\
(\mathrm{p}=0,04)\end{array}$ & $\begin{array}{c}14 \\
(63,6)\end{array}$ \\
\hline
\end{tabular}

* $\mathrm{M}=$ Sexo masculino, $\mathrm{F}=$ Sexo feminino, $\mathrm{PA}=$ pressão arterial, $\mathrm{OB}=$ obesidade, $\mathrm{AF}=$ atividade física, $\mathrm{PAS}=$ pressão arterial sistólica, PAD = pressão arterial diastólica, HAS = hipertensão arterial sistêmica, IMC = índice de massa corporal; CC = circunferência da cintura, $\mathrm{CQ}=$ circunferência do quadril, $\mathrm{MET}=$ equivalente metabólico, $\mathrm{IPAQ}=\mathrm{Questionário} \mathrm{Internacional} \mathrm{de}$ Atividade Física, STROBE = Strengthening the Reporting of Observational Studies in Epidemiology.

arterial em alguns momentos. Moussa et al. ${ }^{42}$, relatam a falta de associação significativa entre razão cintura quadril tanto com PAS, quanto com PAD. Kelishadi et al. ${ }^{34}$, não observaram associação entre peso e PAS, Monyeki et al. ${ }^{11}$ não encontraram associação entre a razão das dobras cutâneas subescapular e triceps (S/T) e PAS, e nem entre nenhuma de sua medidas de obesidade e PAD. Leung et al. ${ }^{49}$ não foram capazes de observar associação entre IMC e HAS, e, por fim,
Hujova et al..$^{50}$ não encontraram associação entre gordura corporal e PA.

Quatro estudos obtiveram através de alguma de suas medidas, associações negativas entre obesidade e $\mathrm{PA}^{34,35,40,46}$.

\section{Atividade física e pressão arterial}

Entre os 16 estudos que relataram os resultados no que diz respeito às associações entre ativi- 
Tabela 3. Estudos que não ilustram associações entre pressão arterial e atividade física e entre pressão arterial e obesidade.

\begin{tabular}{|c|c|c|c|c|}
\hline Referência & $\begin{array}{l}\text { Amostra / } \\
\text { Nação }\end{array}$ & Medidas & Resultados & $\begin{array}{l}\text { Pontuação } \\
\text { STROBE } \\
(\%)\end{array}$ \\
\hline $\begin{array}{l}\text { Moussa et al., } \\
1994^{42}\end{array}$ & $\begin{array}{c}\mathrm{n}=440 \\
\mathrm{M}=240 \\
\mathrm{~F}=200 \\
7 \text { a } 18 \text { anos } \\
\text { Emirados Árabes }\end{array}$ & $\begin{array}{c}\mathrm{PA}=\text { auscultatório } \\
\mathrm{OB}=\mathrm{IMC} \text {, razão } \\
\text { cintura quadril } \\
\mathrm{AF}=\mathrm{NR}\end{array}$ & $\begin{array}{c}\text { Sem associação entre razão } \\
\text { cintura quadril e PAS ( } \mathrm{p}=0,803) \\
\text { Sem associação entre razão } \\
\text { cintura quadril e PAD }(\mathrm{p}=0,648)\end{array}$ & $\begin{array}{l}13,12 \\
(59,6)\end{array}$ \\
\hline $\begin{array}{l}\text { Taylor et al., } \\
2002^{24}\end{array}$ & $\begin{array}{c}\mathrm{n}=82 \\
\mathrm{~F} \\
11 \text { a } 13 \text { anos } \\
\text { Estados Unidos }\end{array}$ & $\begin{array}{c}\text { PA = automático } \\
\text { OB = } \\
\text { circunferência do } \\
\text { abdômen, coxa, } \\
\text { braço, quadril; } \\
\text { dobra cutânea da } \\
\text { panturrilha, coxa, } \\
\text { tríceps, } \\
\text { subescapular e } \\
\text { abdômen; IMC } \\
\text { AF = entrevista } \\
\text { (outro, MET) }\end{array}$ & $\begin{array}{l}\text { Sem associação entre IMC e PAS } \\
\quad(p=N R) \\
\text { Sem associação entre AF e PAS } \\
\quad(p=N R) \\
\text { Sem associação entre IMC e PAD } \\
\quad(p=N R) \\
\text { Sem associação entre AF e PAD } \\
(p=N R)\end{array}$ & $\begin{array}{c}17,5 \\
(79,5)\end{array}$ \\
\hline $\begin{array}{l}\text { Ribeiro et al., } \\
2004^{26}\end{array}$ & $\begin{array}{c}\mathrm{n}=1533 \\
\mathrm{M}= \\
699 \\
\mathrm{~F}=762 \\
8 \text { a } 15 \text { anos } \\
\text { Portugal }\end{array}$ & $\begin{array}{l}\mathrm{PA}=\text { Automático } \\
\mathrm{OB}=\mathrm{IMC} \text {, dobras } \\
\text { cutâneas do tríceps } \\
\text { e subescapular } \\
\text { AF = Questionário } \\
\text { (outro, MET, } \\
\text { calcula o Î́ndice de } \\
\text { Atividade Física) }\end{array}$ & $\begin{array}{l}\text { Masc.: Sem associação entre } \\
\text { percentual de gordura e PAD } \\
\quad(r=0,0921 ; p>0,05)\end{array}$ & $\begin{array}{l}16,75 \\
(76,1)\end{array}$ \\
\hline $\begin{array}{l}\text { Monyeki et al., } \\
2006^{11}\end{array}$ & $\begin{array}{c}\mathrm{n}=1884 \\
\mathrm{M}=967 \\
\mathrm{~F}=917 \\
6 \text { a } 13 \text { anos } \\
\text { África do Sul }\end{array}$ & $\begin{array}{c}\text { PA = automático } \\
\text { OB = IMC, dobras } \\
\text { cutâneas } \\
\text { suprailíacas, } \\
\text { subescapular, } \\
\text { tríceps e bíceps }\end{array}$ & $\begin{array}{l}\text { Sem associação entre S/T e PAS } \\
(\beta=0,001 ; p=0,807) \\
\text { Sem associação entre IMC e PAD } \\
(\beta=0,002 ; p=0,57) \\
\text { Sem associação entre S/T e PAD } \\
(\beta=0,002 ; p=0,76) \\
\text { Sem associação entre S/ST e } \\
\text { PAD }(\beta=0,03 ; p=0,61) \\
\text { Sem associação entre SS/SSBT e } \\
\text { PAD }(\beta=-0,01 ; p=0,54) \\
\text { Sem associação entre somatório } \\
\text { das dobras cutâneas e PAD } \\
(\beta=0,004 ; p=0,86)\end{array}$ & $\begin{array}{c}15 \\
(68,2)\end{array}$ \\
\hline $\begin{array}{l}\text { Kelishadi et al., } \\
2006^{3}\end{array}$ & $\begin{array}{l}\mathrm{n}=21111 \\
\mathrm{M}=10253 \\
\mathrm{~F}=10858 \\
6 \text { a } 18 \text { anos } \\
\text { Irã }\end{array}$ & $\begin{array}{l}\mathrm{PA}=\text { auscultatório } \\
\mathrm{OB}=\mathrm{IMC}, \mathrm{CC}, \\
\text { circunferência do } \\
\text { quadril } \\
\mathrm{AF}=\text { questionário } \\
\text { (MET, boa relação } \\
\text { teste - reteste e } \\
\text { associado } \\
\text { significativamente } \\
\text { com o IPAQ e o } 7 \\
\text { day physical } \\
\text { activity) }\end{array}$ & $\begin{array}{l}\text { Sem associação entre peso e PAS } \\
\qquad(\beta=0,24 ; p=0,656)\end{array}$ & $\begin{array}{c}16 \\
(72,7)\end{array}$ \\
\hline
\end{tabular}


Tabela 3. continuação

\begin{tabular}{|c|c|c|c|c|}
\hline Referência & $\begin{array}{l}\text { Amostra / } \\
\text { Nação }\end{array}$ & Medidas & Resultados & $\begin{array}{l}\text { Pontuação } \\
\text { STROBE } \\
(\%)\end{array}$ \\
\hline $\begin{array}{c}\text { Monego e } \\
\text { Jardim, 2006 }\end{array}$ & $\begin{array}{c}\mathrm{n}=3169 \\
\mathrm{M}=1600 \\
\mathrm{~F}=1569 \\
7 \text { a } 14 \text { anos } \\
\text { Brasil }\end{array}$ & $\begin{array}{c}\mathrm{PA}=\text { auscultatório } \\
\mathrm{OB}=\mathrm{IMC} \\
\mathrm{AF}=\text { entrevista }\end{array}$ & $\begin{array}{l}\text { Sem associação entre AF e níveis } \\
\text { de PA }(p=0,62)\end{array}$ & $\begin{array}{c}15,5 \\
(70,5)\end{array}$ \\
\hline $\begin{array}{l}\text { Singh et al., } \\
2006^{45}\end{array}$ & $\begin{array}{c}\mathrm{n}=510 \\
\mathrm{M}=279 \\
\mathrm{~F}=231 \\
12 \text { a } 18 \text { anos } \\
\quad \text { Índia }\end{array}$ & $\begin{array}{c}\text { PA = automático } \\
\text { OB = IMC } \\
\text { AF = questionário: } \\
\text { Global School- } \\
\text { Based Student } \\
\text { Health Survey } \\
\text { adaptado }\end{array}$ & $\begin{array}{l}\text { Sem associação entre AF e PAS } \\
(p>0,05) \\
\text { Sem associação entre AF e PAD } \\
\quad(p>0,05)\end{array}$ & $\begin{array}{c}13,87 \\
(63)\end{array}$ \\
\hline $\begin{array}{l}\text { Sugyiama et al., } \\
\qquad 2007^{46}\end{array}$ & $\begin{array}{c}\mathrm{n}=4508 \\
\mathrm{M}=50,9 \% \\
\mathrm{~F}=49,1 \% \\
12 \text { a } 19 \text { anos } \\
\text { Estados Unidos }\end{array}$ & $\begin{array}{c}\mathrm{PA}=\text { auscultatório } \\
\mathrm{OB}=\text { IMC } \\
\mathrm{AF}=\text { entrevista } \\
\text { (outro) }\end{array}$ & $\begin{array}{l}\text { Sem associação entre AF moderada } \\
\text { ou vigorosa e PAS ( } \mathrm{p}=\mathrm{NS}) \\
\text { Sem associação entre atividade } \\
\text { física moderada e PAD ( } \mathrm{p}=\mathrm{NS})\end{array}$ & $\begin{array}{l}18,75 \\
(85,2)\end{array}$ \\
\hline $\begin{array}{l}\text { Silva e Lopes, } \\
2008^{27}\end{array}$ & $\begin{array}{c}\mathrm{n}=1570 \\
\mathrm{M}=808 \\
\mathrm{~F}=762 \\
7 \text { a } 12 \text { anos } \\
\text { Brasil }\end{array}$ & $\begin{array}{c}\mathrm{PA}=\text { auscultatório } \\
\mathrm{OB}=\mathrm{IMC} \text {, dobra } \\
\text { cutânea do tríceps } \\
\mathrm{AF}=\text { questionário } \\
\text { Typical Daily } \\
\text { Physical activities } \\
\text { and Meals (DAFA) }\end{array}$ & $\begin{array}{l}\text { Masc.: Sem associação entre AF e } \\
\text { PA elevada }(\mathrm{p}>0,05) \\
\text { Sem associação entre excesso de } \\
\text { gordura corporal e PA elevada } \\
\qquad(\mathrm{p}>0,05) \\
\text { Sem associação entre excesso de } \\
\text { peso e PAD ( } \mathrm{p}>0,05) \\
\text { Sem associação entre excesso de } \\
\text { gordura corporal e PAS (p >0,05) } \\
\text { Sem associação entre excesso de } \\
\text { gordura corporal e PAD ( } \mathrm{p}> \\
\quad 0,05) \\
\text { Femin.: Sem associação entre AF } \\
\text { e PA elevada (p > 0,05) } \\
\text { Sem associação entre excesso de } \\
\text { gordura corporal e PA elevada ( } \mathrm{p} \\
>0,05) \\
\text { Sem associação entre excesso de } \\
\text { gordura corporal e PAS ( } \mathrm{p}>0,05) \\
\text { Sem associação entre excesso de } \\
\text { gordura corporal e PAD } \\
\text { (p }>0,05)\end{array}$ & $\begin{array}{c}17 \\
(77,3)\end{array}$ \\
\hline $\begin{array}{l}\text { Candido et al., } \\
\qquad 2009^{47}\end{array}$ & $\begin{array}{c}\mathrm{n}=780 \\
\text { Ambos os sexos } \\
6 \text { a } 14 \text { anos } \\
\text { Brasil }\end{array}$ & $\begin{array}{c}\mathrm{PA}=\text { auscultatório } \\
\text { e automático } \\
\mathrm{OB}=\mathrm{IMC}, \mathrm{CC} \\
\mathrm{AF}=\text { entrevista } \\
\text { (outro) }\end{array}$ & $\begin{array}{l}\text { Sem associação entre AF e PA } \\
\qquad(p>0,05)\end{array}$ & $\begin{array}{l}16,12 \\
(73,3)\end{array}$ \\
\hline $\begin{array}{l}\text { Obarzanec et al., } \\
2010^{25}\end{array}$ & $\begin{array}{c}\mathrm{n}=2368 \\
\mathrm{~F} \\
10 \text { a } 18 \text { anos } \\
\text { Estados Unidos }\end{array}$ & $\begin{array}{c}\mathrm{PA}=\text { auscultatório } \\
\mathrm{OB}=\mathrm{IMC}, \mathrm{CC} \\
\mathrm{AF}=\text { questionário } \\
\text { (Questionário } \\
\text { modelo de } \\
\text { atividades físicas } \\
\text { habituais) }\end{array}$ & $\begin{array}{c}\text { Sem associação entre AF e } \\
\text { desenvolvimento de HAS } \\
\qquad(\mathrm{p}=0,106)\end{array}$ & $\begin{array}{c}14,75 \\
(67)\end{array}$ \\
\hline
\end{tabular}


Tabela 3. continuação

\begin{tabular}{|c|c|c|c|c|}
\hline Referência & $\begin{array}{c}\text { Amostra / } \\
\text { Nação }\end{array}$ & Medidas & Resultados & $\begin{array}{l}\text { Pontuação } \\
\text { STROBE } \\
(\%)\end{array}$ \\
\hline $\begin{array}{l}\text { Yi-Chun Chou e } \\
\text { Jen-Sheng Pei, } \\
2010^{28}\end{array}$ & $\begin{array}{c}\mathrm{n}=558 \\
\mathrm{M}=284 \\
\mathrm{~F}=274 \\
12 \text { a } 18 \text { anos } \\
\text { Taiwan }\end{array}$ & $\begin{array}{c}\text { PA = automático } \\
\text { OB = IMC, CC } \\
\text { AF = Questionário }\end{array}$ & $\begin{array}{c}\text { Masc.: Sem associação entre AF e } \\
\text { HAS }(\mathrm{p}>0,05) \\
\text { Femin.: Sem associação entre AF } \\
\text { e HAS }(\mathrm{p}>0,05)\end{array}$ & $\begin{array}{l}18,75 \\
(85,2)\end{array}$ \\
\hline $\begin{array}{l}\text { Yoshinaga et al, } \\
2011^{29}\end{array}$ & $\begin{array}{c}\mathrm{n}=755 \\
\mathrm{M}=331 \\
\mathrm{~F}=424 \\
15 \text { a } 18 \text { anos } \\
\text { Japão }\end{array}$ & $\begin{array}{c}\text { PA - automático } \\
\text { OB - IMC, CC } \\
\text { AF - Questionário } \\
\text { (outro) }\end{array}$ & $\begin{array}{c}\text { Masc.: Sem associação entre } \\
\text { tempo total de exercício e PAS } \\
\quad(\mathrm{p}>0,05) \\
\text { Femin.: Sem associação entre AF } \\
\text { extra curriculares e PAS } \\
\quad(\mathrm{p}>0,05) \\
\text { Sem associação entre tempo total } \\
\text { de exercício e PAS }(\mathrm{p}>0,05)\end{array}$ & $\begin{array}{c}17 \\
(77,3)\end{array}$ \\
\hline $\begin{array}{l}\text { Gaya et al., } \\
2011^{48}\end{array}$ & $\begin{array}{l}\mathrm{n}=1075 \\
\mathrm{M}=503 \\
\mathrm{~F}=572 \\
8 \text { a } 17 \text { anos } \\
\text { Portugal }\end{array}$ & $\begin{array}{c}\mathrm{PA}=\text { automático } \\
\mathrm{OB}=\mathrm{IMC} \\
\mathrm{AF}=\text { questionário }\end{array}$ & $\begin{array}{l}\text { Sem associação entre atividades } \\
\text { de competição esportiva e PA alta } \\
(\mathrm{OR}=1,25 ; 0,92-1,70 ; \mathrm{p}=0,14)\end{array}$ & $\begin{array}{l}17,25 \\
(78,4)\end{array}$ \\
\hline $\begin{array}{l}\text { Leung et al., } \\
2011^{49}\end{array}$ & $\begin{array}{c}\mathrm{n}=5901 \\
\mathrm{M}=2938 \\
\mathrm{~F}=2963 \\
\text { Média de } 15,2 \\
\text { anos de idade } \\
\text { China }\end{array}$ & $\begin{array}{c}\mathrm{PA} \text { = automático } \\
\text { OB - IMC } \\
\text { circunferência da } \\
\text { cintura } \\
\text { AF - questionário } \\
\text { (outro) }\end{array}$ & $\begin{array}{c}\text { Sem associação entre IMC e HAS } \\
\qquad(\mathrm{OR}=1,436 ; 0,995-2,021 ; \\
\mathrm{p}=0,053)\end{array}$ & $\begin{array}{l}19,12 \\
(86,9)\end{array}$ \\
\hline $\begin{array}{l}\text { Hujova e } \\
\text { Lesniakova, } \\
2011^{50}\end{array}$ & $\begin{array}{l}\qquad \mathrm{n}=191 \\
\mathrm{M}=97 \\
\mathrm{~F}=94 \\
\text { Idade } 7-11 / 12-18 \\
\text { Eslováquia }\end{array}$ & $\begin{array}{c}\mathrm{PA}=\text { auscultatório } \\
\mathrm{OB}=\mathrm{IMC} \text {, dobras } \\
\text { cutâneas do tríceps } \\
\text { e subescapular, CC, } \\
\text { circunferência do } \\
\text { quadril } \\
\mathrm{AF}=\text { questionário } \\
\text { (outro) }\end{array}$ & $\begin{array}{c}\text { Sem associação entre gordura } \\
\text { corporal e PA }(p>0,05)\end{array}$ & $\begin{array}{c}14 \\
(63,6)\end{array}$ \\
\hline $\begin{array}{l}\text { Aounallah - } \\
\text { Skhiri et al., } \\
2012^{31}\end{array}$ & $\begin{array}{c}\mathrm{n}=2870 \\
\mathrm{M}=1294 \\
\mathrm{~F}=1576 \\
15 \text { a } 19 \text { anos } \\
\text { Tunísia }\end{array}$ & $\begin{array}{c}\mathrm{PA}=\text { auscultatório } \\
\mathrm{OB}=\mathrm{IMC}, \mathrm{CC} \\
\mathrm{AF}=\text { entrevista }\end{array}$ & $\begin{array}{l}\text { Masc.: Sem associação entre AF e } \\
\text { HAS (OR }=2,8 ; 1,1-7 ; \\
\text { p }=0,072) \\
\text { Femin.: Sem associação entre AF } \\
\text { e HAS }(\mathrm{OR}=1,4 ; 0,7-2,9 ; \\
\mathrm{p}=0,075) \\
\text { Sem associação entre CC e HAS } \\
\quad(\mathrm{OR}=1,5 ; 0,7-3,2 ; \mathrm{p}=0,5)\end{array}$ & $\begin{array}{c}17,37 \\
(79)\end{array}$ \\
\hline
\end{tabular}

${ }^{*} \mathrm{M}=$ Sexo masculino, $\mathrm{F}=$ Sexo feminino, $\mathrm{PA}=$ pressão arterial, $\mathrm{OB}=$ obesidade, $\mathrm{AF}=$ atividade física, $\mathrm{PAS}=$ pressão arterial sistólica, $\mathrm{PAD}=$ pressão arterial diastólica, $\mathrm{HAS}=$ hipertensão arterial sistêmica, $\mathrm{IMC}=$ índice de massa corporal; $\mathrm{CC}=$ circunferência da cintura, $\mathrm{S} / \mathrm{T}=$ subescapular/tríceps, S/ST = subescapular/subescapular+tríceps, SS/SSBT = subescapular+suprailíaca/subescapular+suprailíaca+bíceps+tríceps, NR = não relatado MET = equivalente metabólico, NS = não significativo, STROBE = Strengthening the Reporting of Observational Studies in Epidemiology.

dade física e PA, foram identificadas oito estudos com associações negativas, nenhum estudo com associação positiva, e a inexistência de associação foi encontrada em onze momentos (Quadro 1). O número de associações mais uma vez é superior ao número de estudos, devido aos resul- tados algumas vezes discordarem entre os métodos ou gêneros.

Para mensuração da atividade física, sete estudos usaram entrevistas ${ }^{24,31,35,38,44,46,47}$, um estudo fez uso de registros médicos prévios ${ }^{41}$, os demais empregaram questionários. 
Quadro 1. Síntese geral das associações ilustradas pelos estudos.

\begin{tabular}{|c|c|c|c|c|c|}
\hline \multicolumn{2}{|r|}{ Associações positivas } & \multicolumn{2}{|c|}{ Associações negativas } & \multicolumn{2}{|c|}{ Sem associações } \\
\hline PA $\times$ AF & PA $x$ OB & PA $\times$ AF & PA $x$ OB & PA $\times$ AF & PA $x$ OB \\
\hline & Moussa et al., $1994^{42}$ & Paterno, $2003^{43}$ & Kelishadi et al., $2006^{34}$ & Taylor et al., $2002^{24}$ & Moussa et al., $1994^{42}$ \\
\hline & Freedman et al, $1999^{32}$ & $\begin{array}{c}\text { Kelishadi et al., } \\
2006^{34}\end{array}$ & Forrest e Leeds, $2007^{35}$ & $\underset{2006^{44}}{\text { Monego e Jardim, }}$ & Taylor et al., $2002^{24}$ \\
\hline & Paterno, $2003^{43}$ & $\begin{array}{l}\text { Sugyiama et al., } \\
2007^{46}\end{array}$ & Sugyiama et al., $2007^{46}$ & Singh et al., $2006^{45}$ & Ribeiro et al., $2004^{26}$ \\
\hline & Ribeiro et al., $2004^{26}$ & $\begin{array}{l}\text { Griz et al., } \\
2010^{33}\end{array}$ & Burgos et al., $2010^{40}$ & $\begin{array}{l}\text { Sugyiama et al., } \\
2007^{46}\end{array}$ & Monyeki et al., $2006^{11}$ \\
\hline & Li et al., $2005^{36}$ & $\begin{array}{c}\text { Yoshinaga et al., } \\
2011^{29}\end{array}$ & & $\begin{array}{l}\text { Silva e Lopes, } \\
2008^{27}\end{array}$ & Kelishadi et al., $2006^{34}$ \\
\hline & Pileggi et al., $2005^{37}$ & $\begin{array}{l}\text { Gaya et al., } \\
2011^{48}\end{array}$ & & $\begin{array}{l}\text { Candido et al., } \\
2009^{47}\end{array}$ & Silva e Lopes, $2008^{27}$ \\
\hline & Monyeki et al., $2006^{11}$ & $\begin{array}{l}\text { Leung et al., } \\
\quad 2011^{49}\end{array}$ & & $\begin{array}{l}\text { Obarzanec et al., } \\
2010^{25}\end{array}$ & Leung et al., $2011^{49}$ \\
\hline & Ribeiro et al., $2006^{12}$ & $\begin{array}{c}\text { Hujova e } \\
\text { Lesniakova, } \\
2011^{50}\end{array}$ & & $\begin{array}{l}\text { Yi-Chun Chou } \\
\text { e Jen-Sheng Pei, } \\
2010^{28}\end{array}$ & $\begin{array}{l}\text { Hujova e Lesniakova, } \\
2011^{50}\end{array}$ \\
\hline & Kelishadi et al., $2006^{34}$ & & & $\begin{array}{c}\text { Yoshinaga et al., } \\
2011^{29}\end{array}$ & $\begin{array}{l}\text { Aounallah-Skhiri et } \\
\text { al., } 2012^{31}\end{array}$ \\
\hline & Monego e Jardim, $2006^{44}$ & & & Gaya et al., $2011^{48}$ & \\
\hline & Singh et al., $2006^{45}$ & & & $\begin{array}{l}\text { Aounallah- Skhiri } \\
\text { et al., } 2012^{31}\end{array}$ & \\
\hline & Forrest e Leeds, $2007^{35}$ & & & & \\
\hline & Sugyiama et al., $2007^{46}$ & & & & \\
\hline & Silva e Lopes, $2008^{27}$ & & & & \\
\hline & Saha et al., $2008^{38}$ & & & & \\
\hline & Salvadori et al., $2008^{39}$ & & & & \\
\hline & Sporisevic et al., $2009^{23}$ & & & & \\
\hline & Candido et al., $2009^{47}$ & & & & \\
\hline & Obarzanec et al., $2010^{25}$ & & & & \\
\hline & $\begin{array}{c}\text { Yi-Choun Chou e Jen-Sheng } \\
\text { Pei, } 2010^{28}\end{array}$ & & & & \\
\hline & Burgos et al., $2010^{40}$ & & & & \\
\hline & Griz et al., $2010^{33}$ & & & & \\
\hline & Polderman et al., $2011^{30}$ & & & & \\
\hline & Lazorick et al., $2011^{41}$ & & & & \\
\hline & Gaya et al., $2011^{48}$ & & & & \\
\hline & Leung et al., $2011^{49}$ & & & & \\
\hline & Hujova e Lesniakova, $2011^{50}$ & & & & \\
\hline & $\begin{array}{l}\text { Aounallah-Skhiri et al., } \\
2012^{31}\end{array}$ & & & & \\
\hline Total $=0$ & Total $=28$ & Total $=08$ & Total $=04$ & Total $=11$ & Total $=09$ \\
\hline
\end{tabular}

o somatório do total de desfechos é superior ao número de artigos investigados em razão dos resultados alguma vezes divergirem entre os métodos ou entre os gêneros. $\mathrm{PA}=$ pressão arterial; $\mathrm{OB}=$ obesidade; $\mathrm{AF}=$ atividade física 
Ribeiro et al. ${ }^{26}$ usaram um questionário validado anteriormente e calcularam, baseados em equivalentes metabólicos (METs), o índice de atividade física. Aounallah-Skhiri et al. ${ }^{31}$ também comentam que o questionário utilizado pelos entrevistadores foi previamente validado e usam tal instrumento para quantificar os níveis de atividade física de sua amostra, baseado em pontos de corte por METs.

Paterno $^{43}$ utilizou um índice desenvolvido para a aplicação no seu estudo, porém, não faz comentários sobre a validade e nem sobre a reprodutibilidade de tal índice.

Gaya et al. ${ }^{48}$ advogam que o questionário por eles empregado, já foi anteriormente utilizado no mesmo contexto cultural e que mostrou significativa reprodutibilidade.

Kelishadi et al. ${ }^{34}$ esclareceram que o instrumento que utilizaram para avaliação da atividade física apresentou boa relação teste-reteste e se associou significativamente ao International Physical Activity Questionnaire e ao 7 - Day Physical Activity Diary, no seu grupo amostral.

Ainda outros quatro estudos que lançaram mão de questionários identificaram seus instrumentos. Singh et al. ${ }^{45}$ e Griz et al. ${ }^{33}$, utilizaram o Global School-Based Student Health Survey, Silva et al. ${ }^{27}$ usaram o Typical Daily Physical Activities and Meals, e Obarzanec et al. ${ }^{25}$ fizeram uso de um questionário modelo de atividades físicas habituais e ressalvam que tal método é validado.

Dos artigos que empregaram entrevistas, apenas Monego et al. ${ }^{44}$ esclarecem que sua entrevista já foi utilizada em outras pesquisas. Os demais estudos, não identificam e nem comentam a respeito da validação ou reprodutibilidade de seus instrumentos.

\section{Discussão}

\section{Obesidade e pressão arterial}

Os mecanismos propostos para ilustrar a relação entre ganho de peso e HAS dizem respeito a uma maior atividade simpática, aumentando o débito cardíaco e a resistência vascular sistêmica. Tais alterações na hemodinâmica cardiovascular resultariam em mudanças nas cifras pressóricas no sentido de elevá-las.

Uma disfunção vascular caracterizada pela rigidez arterial ou uma dilatação endotelial vascular prejudicada também comprometem os valores pressóricos. O ganho de peso também aumenta a resistência à insulina que por sua vez é fortemente associada à elevação da pressão arterial em jovens ${ }^{19}$.

Embora o embasamento fisiológico pertinentemente ampare a existência de uma ligação entre obesidade e níveis alterados de PA, a literatura nem sempre adula tal perspectiva remetendo assim a situação inconclusiva no que diz respeito a uma visão de causalidade.

A presente revisão foi capaz de identificar a inexistência de associação entre obesidade e PA, bem como, associação negativa entre as referidas variáveis em alguns estudos.

Kelishadi et al. ${ }^{34}$ encontraram associação negativa entre circunferência do quadril e PAD. Porém, considerando que na razão cintura quadril, a circunferência do quadril é o divisor, pode-se imaginar que quanto maior a circunferência do quadril, menor seria o valor da razão cintura quadril. A razão cintura quadril é uma medida valorizada dentro do contexto da avaliação da distribuição da gordura corporal, e valores acima dos seus níveis de corte já foram apontados como preditores de hipertensão e distúrbios cardiometabólicos ${ }^{51}$. Antagonicamente a tal perspectiva, Moussa et al. ${ }^{42}$ não observaram qualquer tipo de associação significante entre tal variável e níveis de PA ( $\mathrm{p}>0,05)$, ainda, Burgos et al. ${ }^{40}$ observaram associação negativa entre razão cintura quadril e PAS e PAD, mesmo que fraca, a referida associação mostrou significância ( $\mathrm{p}<$ $0,05)$ o que conduz a um olhar mais reflexivo sobre tal variável antropométrica.

Tais resultados até agora relatados encaminham a uma reflexão mais profunda e crítica em relação à concepção hegemônica, defendida por alguns autores, no que diz respeito à visão de obesidade como importante fator de risco para doenças cardiovasculares ${ }^{52}$. Não desprezando tal discurso, nem objetivando de forma alguma dissertar apologicamente em relação à obesidade, não se pode desprezar que alguns indícios apontados por nossos resultados mostram que em algumas pesquisas tais associações não acontecem, ou ainda, se mostram negativas, o que leva a repensar cautelosamente sobre o enfoque causal pregado incessantemente sobre tal relação pela literatura científica.

Quanto aos métodos utilizados para avaliar obesidade, todos os estudos empregaram o IMC, sendo também utilizado o índice de Rohrer, circunferência da cintura, circunferência do quadril, peso, razão cintura/quadril, percentual de gordura corporal, somatório de dobras cutâneas e razão entre dobras cutâneas. Interessante salientar que dos estudos que utilizaram dobras 
cutâneas $^{11,12,26,27,35,40,50}$, três fizeram uso das mesmas dobras ${ }^{26,40,50}$, e dois utilizaram apenas a dobra do tríceps ${ }^{27,35}$, os demais não acordam nas dobras cutâneas utilizadas. O presente quadro parece contextualizar a inexistência de uma padronização de protocolos para aferição da composição corporal. Além disso, outras implicações pesam sobre tal medida, como a dificuldade de sua realização em sujeitos obesos, e a difícil reprodutibilidade de tal mensuração ${ }^{53}$. Deve-se também destacar que o IMC foi a única medida utilizada em todos as investigações para tratar obesidade, as outras foram diversas vezes aplicadas para identificar padrões de distribuição de gordura corporal.

Estudos epidemiológicos lançam mão costumeiramente do IMC para investigar obesidade e sobrepeso, dada a sua facilidade de medição ${ }^{43,53}$. Tal índice é usado independente da faixa etária, e encorajado como manobra para avaliação de obesidade em crianças e adolescentes ${ }^{54}$. Sua relação com morbidade e mortalidade, ainda é algumas vezes ratificada ${ }^{55}$. No entanto, associações entre níveis de massa corporal e mortalidade parecem um tanto quanto nebulosas e em algumas situações, ainda, meramente especulativas ${ }^{56}$. Em um grupo de indivíduos hipertensos apontados por Uretsky et al. ${ }^{57}$, por exemplo, a obesidade, classificada pelo IMC, apresentou efeito protetor e não deletério no que diz respeito a condições de morbidade, bem como mortalidade, ilustrando assim um paradoxo se levarmos como via de regra o discurso hegemônico existente sobre a relação sobrepeso, morbidade, mortalidade.

Outras críticas pesam sobre tal índice, já que ele não considera o fracionamento da composição corporal no que se refere à diferenciação entre massa gorda e massa corporal livre de gordura, o que o torna um instrumento capaz de não raramente falhar ao classificar determinadas populações, por exemplo, aquelas com um alto padrão de atividade física ${ }^{58-60}$, além disso, as diferentes curvas de percentil utilizadas para classificar os valores de IMC, muitas vezes dificultam a discussão específica de resultados de diferentes estudos.

Sendo assim, embora a facilidade de coleta do IMC seja indiscutível, deve-se pesar suas vantagens e desvantagens antes de categorizar de forma quase totalitária sua aplicação em estudos que mensuram obesidade. Talvez o desenho do estudo, muitas vezes seja o determinante mais relevante na escolha do IMC como método de análise da obesidade devido mais a viabilidade de tal medida, do que suas qualidades científicas para identificar excesso de gordura corporal.
No entanto, fugindo do âmbito da específica classificação de obesidade, e dos fracionamentos da composição corporal, talvez o IMC tenha eficácia em mensurar excesso de massa corporal e, assim, essa variável seria uma útil ferramenta na associação de IMC com padrões pressóricos em adolescentes. Ma et al. ${ }^{61}$ parearam uma amostra de 13.972 indivíduos entre sete e dezoito anos, por peso e altura, sendo assim, o pareamento também foi efetivo no que diz respeito ao IMC, porém, o percentual de gordura diferia de forma significativa. O mesmo pareamento foi feito com 5.103 indivíduos entre sete e dezoito anos, porém dessa vez com diferença significativa no que diz respeito à circunferência de cintura. Os resultados apontaram que os padrões pressóricos independiam do percentual de gordura e da circunferência de cintura, sendo muito semelhantes e em alguns momentos sem diferença significativa do maior para o menor quartil das citadas variáveis. Os autores levantam a hipótese de que a massa magra e a gordura podem possuir similar impacto na PA de indivíduos dentro de tal contexto etário.

Pode-se também notar uma tendência no interesse em se estudar tais associações na linha temporal, tendo um abrupto aumento no número de estudos publicados sobre o tema na segunda metade da ultima década dos anos investigados (2008-2012). Tal fato pode refletir a crença hegemônica no aumento de peso, e nas conseqüências de tal ocorrência advogadas piamente pela literatura ${ }^{62}$, o que pode direcionar a uma ideia de epidemia e a um constructo de pânico moral. Flegal et al. ${ }^{63}$ abordam a questão conceitual de se associar obesidade ao termo epidemia, e deixam claro que embora a obesidade possa estar em parte dentro do contexto epidêmico, não existe uma definição exata para o que seriam níveis normais de obesidade na população, e pela falta da quantificação de tal dado, seria impreciso e talvez precipitado o julgamento da questão da obesidade como epidemia. Tal termo muitas vezes é empregado de forma metafórica, porém perigosamente interpretado de maneira literal. Antes que se empregue com propriedade o termo epidemia em tal situação, deve-se pesar outras referências literárias que encaminham a reflexão de tal campo, remetendo assim, a incerteza do conhecimento ${ }^{56}$.

\section{Atividade física e pressão arterial}

Fisiologicamente, a atividade física parece se mostrar como tendo interessante impacto na ate- 
nuação das respostas simpáticas, da resistência à insulina e das disfunções endoteliais cardiovasculares. Tal efeito do exercício se caracteriza por diminuição da frequência cardíaca e por consequência do débito cardíaco, e também por uma diminuída resistência vascular sistêmica. Sendo assim, essas alterações na dinâmica cardiovascular podem incidir nos valores da pressão arterial ${ }^{19}$.

A manutenção dos níveis pressóricos elevados cronicamente remodela a parede arterial da carótida a favor de um enrijecimento. Tal característica torna os barorreceptores menos sensíveis, já que os mesmos respondem em favor da homeostase pressórica através da distensão desses vasos ${ }^{64}$. Acredita-se que o exercício físico aumenta a sensibilidade dessas estruturas através de uma maior sensibilização do nervo depressor aórtico, via diminuição da atividade simpática, além de seu efeito direto sobre as respostas autonômicas capazes de antagonizar o enrijecimento arterial como já foi anteriormente exposto ${ }^{65}$.

Interessantemente, embora o presente discurso de mecanismos fisiológicos enrobusteça a credibilidade acerca do impacto da atividade física sobre padrões pressóricos, a literatura não é consensual em relação a tais respostas no que diz respeito aos adolescentes ${ }^{2}$. Os resultados da presente revisão parecem ratificar tal conflito literário, não evidenciando de forma majoritária, indícios de uma associação negativa entre as respectivas variáveis como sugere o pensamento hegemônico.

Os resultados também sublinham a inexistência de uma padronização no que tange aos métodos de mensuração do nível de atividade física, com um grande número de investigações sequer informando a respeito da validade ou reprodutibilidade de seus instrumentos para tal coleta.

Validar medidas subjetivas de atividade física, ainda é uma questão que se defronta contra outro problema ainda maior, que diz respeito à inexistência de um padrão ouro adequado e consensual, com o qual questionários e entrevistas pudessem ser confrontados para análise de suas respectivas validades ${ }^{66}$.

A discussão literária entre tais estudos se torna difícil tarefa. A falta de padronização de instrumentos de medida em relação à atividade física, e a subjetividade ilustrada em tais medidas, sublinham tal situação ${ }^{67}$.

Também vale destacar, que os estudos mensuram atividade física e sedentarismo muitas vezes de formas diferentes, o que leva a reflexão sobre o conceito de sedentarismo. Seria tal vari- ável um nível insatisfatório de atividade física? Seria tal variável uma medida associada a certos hábitos de vida? Se conceituar sedentarismo é difícil, atribuir relações causais, na esfera da saúde, a tal termo é ainda mais complexo. A falta de um conceito preciso e científico para o termo sedentarismo, talvez seja a gênese de discursos tão dicotômicos.

Mais uma vez, foi observada uma tendência temporal de aumento quantitativo nesse tipo de investigação na segunda metade da ultima década dos anos cobertos pela presente revisão (20082012). Embora uma crença no aumento do sedentarismo devido aos avanços e facilidades tecnológicas da sociedade contemporânea ${ }^{68}$ possa ser o pressuposto estímulo para tal acontecimento, espera-se que baseado nas fragilidades conceituais e mensuráveis do que seja sedentário, já antes discutido, tal questão seja objeto de reflexão e não de rotulação.

Tratar de sedentarismo e de mudanças de hábito de vida é algo muito mais complexo do que pode parecer, tal discussão extrapola as esferas biológicas, quantitativas e individuais. Limitar-se a tais esferas seria uma forma cética e reducionista de apreciar tais fenômenos. Características qualitativas e coletivas deveriam ser levadas em consideração em tal contexto, esclarecendo assim que a mudança de tais hábitos pode ser uma tarefa não tão simples. Fatores psicossociais ou demográficos deveriam ser levados em consideração toda vez que tal discussão fosse objeto de reflexão. Tais fatores, muitas vezes desconsiderados, parecem exercer influência sobre a prática de atividade física, ratificando-se que, em adolescentes, a rede social merece especial atenção no que toca a influência no estilo de vida ${ }^{69}$.

\section{Pressão arterial}

Levando em conta que o nível pressórico é o desfecho de interesse da presente revisão, tornase relevante pontuar algumas considerações pertinentes a tal variável.

Dezesseis estudos utilizaram o método auscultatório de aferição da $\mathrm{PA}^{23,25,27,31-35,37,38,40,42-}$ ${ }^{44,46,50}$, nove artigos mensuraram a PA com o método automático ${ }^{11,24,26,28,29,30,45,48,49}$, Salvadori et al. ${ }^{39}$ realizaram uma medida manual e duas em aparelhos automáticos, considerando como valor pressórico para seu estudo a média das três. Candido et al. ${ }^{47}$ lançaram mão da média de três medidas realizadas em aparelho automático. Quando tal média excedia o valor do percentil 90 para classificação da PA, eles então aplicavam o 
método auscultatório. Um estudo utilizou registros médicos prévios ${ }^{41}$, Ribeiro et al. ${ }^{12}$ e Forrest e Leed $^{35}$ não descrevem claramente em seus métodos qual tipo de instrumento usou para aferir a PA.

Ainda que a maioria dos estudos tenha utilizado o método auscultatório, os nove estudos que fizeram suas mensurações com aparelhos automáticos, se localizam na ultima década dos anos da busca da presente revisão - 2002/2012 , tal constatação pode sugerir um crescente apreço por tal método nos últimos tempos. Esse tipo de medida tem algumas relevantes vantagens tal como a eliminação do viés do observador. Vale mencionar ainda que o método auscultatório pode ter fatores complicadores em determinadas populações, como por exemplo, em crianças, quando o desaparecimento dos sons de Korotkoff, pode não ocorrer ${ }^{70}$, fazendo com que o observador tenha que possuir bastante sensibilidade na identificação das mudanças de fases dos sons de Korotkoff. A praticidade dos instrumentos automáticos, principalmente quando se trata de estudos epidemiológicos de amostras representativas, que na maioria das vezes são compostos por um $n$ amostral de tamanho considerável, também evidencia a interessante estratégia do uso de tais aparelhos.

Quanto à forma de classificação da PA, as recomendações são que se leve em consideração o percentil para altura, idade e sexo específico em indivíduos até 17 anos $^{3}$, porém, cinco artigos $28,42,43,45,50$, usaram valores de corte bruto para classificar sua amostra em relação aos níveis pressóricos, mesmo tendo em seu grupo amostral sujeitos com menos de 17 anos. Gaya et al. ${ }^{48}$ dividiram os sujeitos de seu estudo em quartis com base nos valores pressóricos, categorizando como hipertensos indivíduos no ultimo quartil, e os demais, como normotensos. E ainda, Forrest e Leeds ${ }^{35} \mathrm{e}$ Saha et al..$^{38}$ não esclarecem a respeito dos meios utilizados para classificar seu grupo amostral no que diz respeito às cifras pressóricas.

Apenas dois estudos ${ }^{23,49}$ mediram a PA em mais de uma ocasião. A literatura aponta, que a medida da PA em dias distintos, pode trazer valores mais fidedignos da citada variável, levando, muitas vezes, à redução quantitativa da prevalência de $\mathrm{HAS}^{70}$. Talvez a dificuldade de uma amostra bem comprometida, assim como, muitas vezes, o difícil acesso à determinada população, façam com que a medida da pressão arterial em uma única ocasião seja a estratégia mais viável, embora isso possa comprometer os achados.

\section{Medidas associativas}

Local de destaque em estudos que envolvem medidas de associação é ocupado pela escolha dos testes estatísticos, que muitas vezes, se feito de forma equivocada, pode gerar ou até mesmo sustentar hipóteses que descaracterizam a realidade quantitativa das variáveis investigadas.

Dentro dessa linha de raciocínio, Freedman et al. ${ }^{32}$ apontam que os testes de correlação, muitas vezes utilizados entre exposição e desfecho, como mostra a presente revisão, não refletem medidas epidemiológicas como riscos ou chances, que podem não ter um caráter linear.

Ainda, entre as medidas de associação de cunho epidemiológico, alguns cuidados devem ser tomados de acordo com o delineamento investigativo.

Observa-se nesta revisão a predominância de estudos transversais, e nos mesmos na grande maioria, a utilização do Odds Ratio. Tal medida, em casos que o desfecho não é raro, pode superestimar os valores associativos. Porém, quando a prevalência da doença é pequena na população estudada, a Odds Ratio parece se assemelhar muito com a Razão de Prevalência ${ }^{71}$. Assim sendo, acredita-se que, em eventos raros, ambas as medidas refletem resultados semelhantes, e quando o evento é comum ocorre uma superestimação por parte da Odds Ratio, portanto, parece prudente a escolha da Razão de Prevalência como medida associativa em estudos transversais, porém essa reflexão não se exemplifica na prática atual das análises estatísticas.

A solução de tal problema não é simples. Pois se a prevalência do evento pode ter influência sobre a medida estatística utilizada, é de suma importância o conhecimento de tal dado antes da manipulação numérica das estimativas amostrais. Pois bem, a maioria dos estudos não discursa a respeito do cálculo amostral utilizado para compor a amostra, e, assim, sem uma informação probabilística, se faz inviável a rotulação de desfecho raro ou desfecho comum.

Outro problema estatístico é a criação de pontos de corte ou índices internos aos próprios estudos $^{32}$, tal fato impossibilita muitas vezes a comparação entre investigações.

\section{Considerações finais}

Um pensamento hegemônico parece nortear subliminarmente o conceito contemporâneo da 
apreciação entre certas exposições e desfechos relacionados à saúde.

$\mathrm{Na}$ presente revisão, teve-se a obesidade e a atividade física como tais exposições, e os níveis pressóricos como desfecho. O paradigma dominante aprecia tais ocorrências na direção de uma visão deveras causal entre baixos níveis de atividade e valores pressóricos anormais, e obesidade e valores pressóricos anormais. Via de regra, tal discurso, engenhosamente replicado e ratificado, merece olhares mais críticos e questionadores, antes da passiva aceitação de tais informações.

Pôde-se identificar na presente busca, que a maioria dos artigos que estudam tais relações tem caráter transversal, apenas uma investigação ${ }^{25}$ conduziu um desenho longitudinal. Embora tal estudo tenha restringido sua amostra ao sexo feminino, permitindo poucas extrapolações dentro do presente contexto investigativo, a relevância que tal delineamento denota no parecer de associações, merece destaque na apreciação de seus resultados. Infelizmente, talvez pela dificuldade de seleção e seguimento de uma coorte por um longo período de tempo, a reduzida quantidade de estudos com esse caráter impossibilita uma maior reflexão sobre os achados que o método poderia revelar, contabilizando muito pouco quantitativamente, tendo em vista a maioria avassaladora de estudos transversais.

Estudos transversais não atendem ao critério da temporalidade. Segundo tal critério, para que haja de fato causalidade, a exposição deve sempre preceder o desfecho, e no caso de estudos transversais esse tipo de informação se faz inviável, visto que dados sobre exposição e desfecho são coletados em único momento. Tal critério, dentre os de Hill, é o único considerado sine qua non na análise de causa e efeito ${ }^{72}$.

No que tange aos discursos inseridos no campo da saúde em relação às associações, que se destaque a necessidade de uma reflexão mais critica antes da aceitação do ditado majoritário sobre a premissa de causa e efeito. Em que pesem as considerações literárias que discorrem suas palavras na defesa da ligação entre exposições e desfechos, tal como obesidade e doenças crôni$\mathrm{cas}^{62}$, outros olhares direcionam a reflexões descontextualizadas das matrizes biológicas, porém não menos importantes, e que dizem respeito a quanto de interesse existe por trás da disseminação maciça de tais informações ${ }^{56}$.

Também é valido destacar a diversidade e a fragilidade dos instrumentos utilizados para a mensuração das variáveis em questão. A medida de níveis de atividade física parece por vezes se mostrar metodologicamente ineficiente ${ }^{73}$. A identificação de instrumentos menos subjetivos e mais padronizados poderia facilitar futuras conclusões em relação às referidas associações. Porém, a comunidade cientifica parece mais interessada na inserção de novos instrumentos do que na padronização das medidas. Farias Junior et al. ${ }^{66}$, em uma revisão sistemática sobre instrumentos self report para medidas de atividade física, identificaram 52 instrumentos diferentes, porém apenas 11 foram testados mais de uma vez. Tal fato parece transparecer um esforço maior por parte dos pesquisadores em inserir instrumentos no devido campo de intervenção, do que em testar, trabalhar, aperfeiçoar e quem sabe enfim, em algum momento, identificar alguma forma de padronização razoavelmente aceitável.

Complexidades de ordem operacional existentes no diagnóstico de determinadas variáveis como a $\mathrm{HAS}^{74}$, também sublinham faces pontuais de fragilidades no discurso hegemônico sobre visões casuísticas.

Observam-se na presente revisão, algumas associações negativas entre obesidade e PA, bem como a inexistência de associação em alguns momentos. Em relação à atividade física e PA, também foi evidenciado em algumas investigações levantadas a falta de associação. Tal quadro reforça a necessidade de se refletir sobre o enfoque causal que se tenta atribuir às relações entre exposição e desfecho.

Por fim, que se questione a aceitação passiva de paradigmas pré-existentes, diante da valorização de um olhar mais critico em relação às informações e valores inseridos no campo da saúde. 


\section{Colaboradores}

VG Corrêa Neto participou da elaboração dos métodos, busca e revisão dos estudos, análise dos resultados, revisão crítica e da redação do manuscrito. A Palma participou da elaboração dos métodos, busca e revisão dos estudos, revisão e inclusão de modificações à versão final do manuscrito.

\section{Agradecimentos}

À Coordenação de Aperfeiçoamento do Pessoal de Nível Superior - CAPES, pela bolsa de Mestrado de Victor Gonçalves Corrêa Neto e ao Conselho Nacional de Desenvolvimento Científico e Tecnológico - CNPQ, pela bolsa de produtividade de Alexandre Palma.

\section{Referências}

1. Chobanian AV, Bakris GL, Black HR, Cushman WC, Green LA, Izzo JL, Jones DW, Materson BJ, Oparil S, Wright JT, Roccella EJ. Seventh report of the joint national committee on prevention, detection, evaluation, and treatment of high blood pressure. Hypertension 2003; 42(6):1206-1252.

2. Pescatello LS, Franklin BA, Fagard R, Farquhar WB, Kelley GA, Ray CH. Exercise and hypertension. Med Sci Sport Exer 2004; 36(3):533-553.

3. Pickering TG, Hall JE, Appel LJ, Falkner BE, Graves J, Hill MN, Jones DW, Kurtz T, Sheps SG, Roccella EJ. Recommendations for blood pressure measurement in humans and experimental animals - Part 1: Blood pressure measurement in humans. Hypertension $2005 ; 45(1): 142-161$.

4. Lessa I. Hipertensão arterial sistêmica no Brasil: tendência temporal. Cad Saude Publica 2010; 26(8):1470.

5. Hajjar I, Kotchen TA. Trends in prevalence, awareness, treatment and control of hypertension in the United States, 1988-2000. JAMA 2003; 290(2):199-206.

6. Muntner P, He J, Cutler JA, Wildman RP, Whelton PK. Trends in blood pressure among child and adolescents. JAMA 2004; 291(17):2107-2113.

7. Lauer RM, Clarke WR. Childhood risk factors for high adult blood pressure: the Muscatine study. Pediatrics 1989; 84(4):633-641.

8. Lurbe E, Cifkova R, Crickshank JK, Dillon MJ, Ferreira I, Invitti C, Kuznetsova T, Laurent S, Mancia G, Morales-Oliva F, Rascher W, Redon J, Schaefer F, Seeman T, Stergiou G, Wuhl E, Zanchetti. Management of high blood pressure in children and adolescents: recommendations of the European Society of Hypertension. J Hypertens 2009; 27(9):1719-1742.

9. Serdula MK, Ivery D, Coates RJ, Freedman DS, Williamson DF, Byers T. Do obese children become obese adults? A review of the literature. Prev Med 1993; 22(2):167-177.

10. Sorof JM, Lai D, Turner J, Poffenbarger T, Portman RJ. Overweight, ethnicity, and the prevalence of hypertension in school-aged children. Pediatrics 2004; 113(3 Pt 1):475-482.

11. Monyeki KD, Kemper HCG, Makgae PJ. The association of fat patterning with blood pressure in rural south African children: the Ellisras longitudinal growth and health study. Int J Epidemiol 2006; 35(1):114-120.

12. Ribeiro RQC, Lotufo PA, Lamounier JA, Oliveira RG, Soares JF, Botter DA. Additional cardiovascular risk factors associated with excess weigth in children and adolescents. The Belo Horizonte study. Arq Bras Cardiol 2006; 86(6):406-415.

13. Aglony IM, Arnaiz GP, Acevedo BM, Barja YS, Márquez US, Guzmán AB, Berrios CX. Perfil de presión arterial e historia familiar de hipertension em niños escolares sanos de Santiago de Chile. Rev Med Chil 2009; 137(1):39-45.

14. Sorof J, Daniels S. Obesity hypertension in children: a problem of epidemic proportions. Hypertension 2002; 40(4):441-447.

15. Moore WE, Stephens A, Wilson T, Wilson W, Eichner JE. Body mass index and blood pressure screening in a rural public school system: the health kids project. Prev Chronic Dis 2006; 3(4):1-10. 
16. Guimarães ICB, Almeida AM, Santos AS, Barbosa DBV, Guimarães AC. Blood pressure: Effect of body mass index and of waist circumference on adolescents. Arq Bras Cardiol 2008; 90(6):393-399.

17. Stray-Pedersen M, Helsing RM, Gibbons L, Cormick G, Holmen TL, Vik T, Belizán JM. Weight status and hypertension among adolescents girls in Argentina and Norway: Data from the ENNyS and HUNT studies. BMC Public Health 2009; 9:398.

18. Tremblay MS, Willms JD. Is the Canadian childhood obesity epidemic related to physical inactivity? Int J Obes Relat Metab Disord 2003; 27(9):11001105.

19. Torrance B, McGuire KA, Lewanczuk R, McGavock J. Overweight, physical activity and high blood pressure in children: a review of the literature. Vasc Health Risk Manag 2007; 3(1):139-149.

20. Dickinson HO, Mason JM, Nicolson DJ, Campbell F, Beyer FR, Cook JV, Williams B, Ford GA. Lifestyle interventions to reduce raised blood pressure: a systematic review of randomized controlled trials. J Hypertens 2006; 24(2):215-233.

21. Elm EV, Altman DG, Egger M, Pocock SJ, Gotzsche PC, Vandenbroucke JP. The Strengthening the Reporting of Observational Studies in Epidemiology (STROBE) Statement: Guidelines for Reporting Observational Studies. J Clin Epidemiol 2008; 61(4):344-349.

22. Mendes KG, Theodoro H, Rodrigues AD, Olinto MTA. Prevalência de síndrome metabólica e seus componentes na transição menopáusica: uma revisão sistemática. Cad Saude Publica 2012; 28(8):14231437.

23. Sporisevic L, Krzelj V, Bajraktarevic A, Jahic E. Evaluation of cardiovascular risk in school children. Bosn J Basic Med Sci 2009; 9(3):182-186.

24. Taylor WC, Chan W, Cummings SS, Simons-Morton BG, Day S, Sangi-Haghpeykar H, Pivarnik JS, Mueller WH, Detry MA, Wei II, Johnson-Masotti AP, Hsu HA. Health Growth: Project description and baseline findings. Ethn Dis 2002; 12(4):567-577.

25. Obarzanek E, Wu CO, Cutler JA, Kavey REW, Pearson GD, Daniels SR. Prevalence and incidence of hypertension in adolescents girls. J Pediatr 2010; 157(3):461-467.

26. Ribeiro JC, Guerra S, Oliveira J, Teixeira-Pinto A, Twisk JWR, Duarte JA, Mota J. Physical activity and biological risk factors clustering in pediatric population. Prev Med 2004; 39(3):596-601.

27. Silva KS, Lopes AS. Excess weight, arterial pressure and physical activity in commuting to school: correlations. Arq Bras Cardiol 2008; 91(2):84-91.

28. Yi-Chun Chou MA, Jen-Sheng Pei MA. Risk factors of adolescent obesity in Taiwan and its association in physical activity, blood pressure and waist circumference. Asian J Sports Med 2010; 1(4):214-222

29. Yoshinaga M, Hatake S, Tachikawa T, Shinomiya M, Miyazaki A, Takahashi H. Impact of lifestyles of adolescents and their parents on cardiovascular risk factors in adolescents. J Atheros Thromb 2011; 18(11):981-990.
30. Polderman J, Gurgel RQ, Barreto-Filho JAS, Roelofs R, Ramos REO, Munter JS, Wendte JF, Agyemang C. Blood pressure and BMI in adolescents in Aracaju, Brazil. Public Health Nutr 2011; 14(6):1064-1070.

31. Aounallah-Skhiri H, El Ati J, Traissac P, Ben Romdhane H, Eymard-Duvernay S, Delpeuch F, Achour N, Maire B. Blood pressure and associated factors in a north African adolescent population. A national cross-sectional study in Tunisia. BMC Public Health 2012; 12:98.

32. Freedman DS, Dietz WH, Srinivasan SR, Berenson GS. The relation of overweight to cardiovascular risk factors among children and adolescents: The Bogalusa Heart Study. Pediatrics 1999; $103(6 \mathrm{Pt}$ 1):1175-1182.

33. Griz LHM, Viégas M, Barros M, Griz AL, Freese E, Bandeira F. Prevalence of central obesity in a large sample of adolescents from public schools in Recife, Brazil. Arq Bras Endocrinol Metabol 2010; 54(7):607611.

34. Kelishadi R, Ardalan G, Gheiratmand R, Majdzadeh R, Delavari A, Heshmat R, Gouya MM, Razaghi EM, Motaghian M, Mokhtari MR, Barekati H, Arabi MS. Blood pressure and its influencing factors in a national representative sample of Iranian children and adolescents: the CASPIAN Study. Eur J Cardiovasc Prev Rehabil 2006; 13(6):956-963.

35. Forrest KYZ, Leeds MJ. Prevalence and associated factors of overweight among Mexican-American adolescents. J Am Diet Assoc 2007; 107(10):17971800.

36. Li YP, Yang XG, Zhai FY, Piao JH, Zhao WH, Zhang J, Ma GS. Disease risks of childhood obesity in China. Biomed Environ Sci 2005; 18(6):401-410.

37. Pileggi C, Carbone V, Nobile CGA, Pavia M. Blood pressure and related cardiovascular disease risk factors in 6-18 year-old students in Italy. J Paediatr Child Health 2005; 41(7):347-352.

38. Saha I, Paul B, Dasgupta A, Ghosh P. Variations of adolescent blood pressure by Multifactorial Analysis in an urban slum of Kolkata. J Indian Med Assoc 2008; 106(9):571-572,574,578.

39. Salvadori M, Sontrop JM, Garg AX, Truong J, Suri RS, Mahmud FH, Macnab JJ, Clark WF. Elevated blood pressure in relation to overweight and obesity among children in a rural Canadian Community. Pediatrics 2008; 122(4):e821-e837.

40. Burgos MS, Reuter CP, Burgos LT, Pohl HH, Pauli LTS, Horta JA, Reckziegel MB, Franke SI, Prá D, Camargo M. Comparison analysis of blood pressure, obesity and cardio-respiratory fitness in schoolchildren. Arq Bras Cardiol 2010; 94(6):739-744.

41. Lazorick S, Peaker B, Perrin EM, Schmid D, Pennington T, Yow A, DuBard CA. Prevention and treatment of childhood obesity: care received by a state Medicaid population. Clin Pediatr (Phila) 2011; 50(9):816-826.

42. Moussa MAA, Skaik MB, Selwanes SB, Yaghy OY, Bin-Othman SA. Contribution of body fat and fat pattern to blood pressure level in school children. Eur J Clin Nutr 1994; 48(8):587-590

43. Paterno CA. Coronary Risk Factors in adolescence. The FRICELA study. Rev Esp Cardiol 2003; 56(5):452458. 
44. Monego ET, Jardim PCBV. Determinants of risk of cardiovascular diseases in schoolchildren. Arq Bras Cardiol 2006; 87(1):37-45.

45. Singh AK, Maheshwari A, Sharma N, Anand K. Lifestyle associated risk factors in adolescents. Indian J Pediatr 2006; 73(10):55-60.

46. Sugyiama T, Xie D, Graham-Maar RC, Inoue K, Kobayashi Y, Stettler N. Dietary and lifestyle factors associated with blood pressure among U.S. adolescents. J Adolesc Health 2007; 40(2):166-172.

47. Candido APC, Benedetto R, Castro APP, Carmo JS, Nicolato RLC, Nascimento-Neto RM, Freitas RN, Freitas SN, Caiaffa WT, Machado-Coelho GL. Cardiovascular risk factors in children and adolescents living in an urban area of Southeast of Brazil: Ouro Preto Study. Eur J Pediatr 2009; 168(11):1373-1382.

48. Gaya AR, Silva P, Martins C, Gaya A, Ribeiro JC, Mota J. Association of leisure time physical activity and sports competition activities with high blood pressure levels: study carried out in a sample of Portuguese children and adolescents. Child Care Health Dev 2011; 37(3):329-334.

49. Leung LCK, Sung RYT, So HK, Wong SN, Lee KW, Lee KP, Yam MC, Li SP, Yuen SF, Chim S, Chan KK, Luk D. Prevalence and risk factors for hypertension in Hong Kong Chinese adolescents: waist circumference predicts hypertension, exercise decrease risk. Arch Dis Child 2011; 96(9):804-809.

50. Hujova Z, Lesniakova M. Anthropometric risk factors of atherosclerosis: differences between urban and rural East-Slovakian children and adolescents. Bratisl Lek Lyst 2011; 112(9):491-496.

51. Pereira RA, Sichieri R, Marins VMR. Razão cintura/quadril como preditor de hipertensão arterial. Cad Saude Publica 1999; 15(2):333-344.

52. Donnelly JE, Blair SN, Jakicic JM, Manore MM, Rankin JW, Smith BK. Appropriate physical activity intervention strategies for weight loss and prevention of weight regain for adults. Med Sci Sport Exer 2009; 41(7):459-471.

53. Wells JCK, Cole TJ, Treleaven P. Age - variability in body shape associated with excess weight: the UK national Sizing Survey. Obesity 2008; 16(2):435-441.

54. Dietz WH, Bellizze MC. Introduction: the use of body mass index to assess obesity in children. Am J Clin Nutr 1999; 70(1):123s-125s.

55. Lahti-Koski M, Gill T. Defining childhood obesity. Pediatr Adolesc Med 2004; 9:1-19.

56. Campos P, Saguy A, Ernsberger P, Oliver E, Gaesser G. The epidemiology of overweight and obesity: public health crisis or moral panic? Int J Epidemiol 2006; 35(1):55-60.

57. Uretsky S, Messerli FH, Bangalore S, Champion A, Cooper-Dehoff RM, Zhou Q, Pepine CJ. Obesity paradox in patients with hypertension and coronary artery disease. Am J Med 2007; 120(10):863-870.

58. Garn SM, Leonard WR, Hawthorne VM. Three limitations of the body mass index. Am J Clin Nutr 1986; 44(6):996-997.

59. Yao M, Roberts SB, Ma G, Pan H, Mccrory MA. Field methods for body composition assessment are valid in healthy Chinese adults. J Nutr 2002; 132(2):310-317.
60. Witt KA, Bush EA. College athletes with an elevated body mass index often have a high upper arm muscle area, but not elevated triceps and subscapular skinfolds. J Am Diet Assoc 2005; 105(4):599-602.

61. Ma J, Wang Z, Dong B, Song Y, Hu P, Zhang B. Body fat and blood pressure: comparison of blood pressure measurements in Chinese children with different body fat levels. Br J Nutr 2012; 108(9):1672-1677.

62. Kim S, Popkin BM. Commentary: Understanding the epidemiology of overweight and obesity - a real global public health concern. Int J Epidemiol 2006; 35(1):60-67.

63. Flegal KM. Commentary: The epidemic of obesity what's in a name. Int J Epidemiol 2006; 35(1):72-74.

64. Honzíková N, Fiser B. Baroreflex and essential hypertension in adolescents. Physiol Res 2009; 58(5):605-612.

65. Negrão CE, Rondon MUPB. Exercício físico, hipertensão e controle barorreflexo da pressão arterial. Rev Bras Hipertens 2001; 8(1):89-95.

66. Faria Júnior JC, Lopes Ada S, Florindo AA, Hallal PC. Validade e reprodutibilidade dos instrumentos de medida da atividade física do tipo self-report em adolescentes: uma revisão sistemática. Cad Saude Publica 2010; 26(9):1669-1691.

67. Sirard JS, Pate RR. Physical activity assessment in children and adolescents. Sports Med 2001; 31(6):439454.

68. Hill JO, Peters JC. Environmental contributions to the obesity epidemic. Science 1998; 280(5368):13711374.

69. Palma A, Vilaça MM. O sedentarismo da epidemiologia. Rev Bras Ciênc Esporte 2010; 31(2):105-119.

70. Chiolero A, Bovet P, Paredis G, Paccaud F. Has blood pressure increased in children in response to the obesity epidemic? Pediatrics 2007; 119(3):544-553.

71. Francisco PMS, Donalisio MR, Barros MBA, Cesar CLG, Carandina L, Goldbaum M. Medidas de associação em estudo transversal com delineamento complexo: razão de chances e razão de prevalências. Rev Bras Epidemiol 2008; 11(3):347-355.

72. Medronho RM, Bloch KV, Luiz RR, Werneck GL. Epidemiologia. 2a Edição. Rio de Janeiro: Atheneu; 2009.

73. Romero A, Slater B, Florindo AA, Latorre MRDO, Cezar C, Silva MV. Determinantes do índice de massa corporal em adolescentes de escolas públicas de Piracicaba, São Paulo. Cien Saude Colet 2010; 15(1):141-149.

74. Silva DAS, Lima RLA, Dellagrana RLA, Bacil EDA, Rech CR. Pressão arterial elevada em adolescentes: prevalência e fatores associados. Cien Saude Colet 2013; 18(11):3391-3400.

Artigo apresentado em 08/09/2012

Aprovado em 12/11/2012

Versão final apresentada em 22/11/2012 